Supporting Information

\title{
Nitroxide Radical Polymer-Solvent Interactions and Solubility Parameter Determination
}

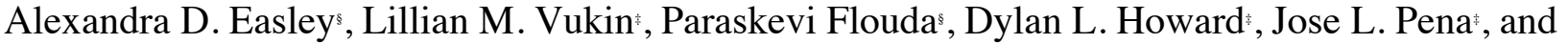
Jodie L. Lutkenhaus s.”.

$\S$ Department of Materials Science and Engineering, Texas A\&M University, College Station, TX 77843, USA

${ }^{\ddagger}$ Artie McFerrin Department of Chemical Engineering, Texas A\&M University, College Station, TX 77843, USA

*Corresponding author: jodie.lutkenhaus@tamu.edu
} 
Table S1. PTMA-based electrodes, electrolyte utilized, and cycling stability of the cell from literature. See ref. 1 for a complete review with additional examples.

\begin{tabular}{|c|c|c|c|}
\hline Electrode System & Electrolyte (v/v) & Capacity Fade/Dissolution & Reference \\
\hline $\begin{array}{l}10 \% \text { PTMA with graphite and } \\
\text { binding powder }\end{array}$ & $\begin{array}{l}1 \mathrm{MLiPF}_{6} \text { in } \\
\mathrm{EC} / \mathrm{DEC}_{(3 / 7)}\end{array}$ & $\begin{array}{l}\text { Initial: } 77 \mathrm{~mA} \cdot \mathrm{h} \cdot \mathrm{g}^{-1} \\
\text { "No significant fade after } 500 \text { cycles" }\end{array}$ & 2 \\
\hline $\begin{array}{l}\text { PTMA:acetylene black:PVDF } \\
=77: 15: 8 \mathrm{wt} \%\end{array}$ & $\begin{array}{l}1 \mathrm{MLiPF}_{6} \text { in } \\
\mathrm{EC} / \mathrm{DMC}(1 / 1)\end{array}$ & $\begin{array}{l}\text { Initial: } 78.4 \mathrm{~mA} \cdot \mathrm{h} \cdot \mathrm{g}^{-1} \\
98 \% \text { of the initial capacity was } \\
\text { obtained after } 100 \text { cycles }\end{array}$ & 3 \\
\hline $\begin{array}{l}\text { PTMA:graphite:PVDF }= \\
\text { 10:80:10 wt } \%\end{array}$ & $\begin{array}{l}0.1 \mathrm{M} \mathrm{TBABF}_{4} \text { in } \\
\mathrm{DCM} \text { or } \mathrm{ACN}\end{array}$ & $\begin{array}{l}\text { Initial: } 77 \mathrm{~mA} \cdot \mathrm{h} \cdot \mathrm{g}^{-1} \\
\text { "no significant deterioration in the } \\
\text { capacity was observed up to } 1000 \\
\text { cycles" }\end{array}$ & 4 \\
\hline $\begin{array}{l}\text { PTMA:VGCF:CMC:PTFE } \\
=50: 45: 4: 1 \mathrm{wt} \%\end{array}$ & $\begin{array}{l}1 \mathrm{M} \mathrm{LiPF}_{6} \text { in } \\
\mathrm{EC} / \mathrm{DEC}_{(3 / 7)}\end{array}$ & $\begin{array}{l}\text { Initial: } 147 \mathrm{~mA} \cdot \mathrm{h} \cdot \mathrm{g}^{-1} \\
82 \% \text { of the initial capacity remained } \\
\text { after } 100 \text { cycles }\end{array}$ & 5 \\
\hline $\begin{array}{l}\text { PTMA:VGCF:PVDF } \\
=30: 60: 10 \mathrm{wt} \%\end{array}$ & $\begin{array}{l}1 \mathrm{M} \mathrm{LiPF}_{6} \text { in } \\
\mathrm{EC} / \mathrm{DEC}_{(3 / 7)}\end{array}$ & $\begin{array}{l}\text { Initial: } 100^{\mathrm{a}} \mathrm{mA} \cdot \mathrm{h} \cdot \mathrm{g}^{-1} \\
89 \% \text { of the initial capacity remained } \\
\text { after } 1000 \text { cycles }\end{array}$ & 6 \\
\hline $\begin{array}{l}\text { PTMA:VGCF:CMC:PTFE } \\
=50: 45: 4: 1 \mathrm{wt} \%\end{array}$ & $\begin{array}{l}1 \mathrm{M} \mathrm{LiPF}_{6} \text { in } \\
\mathrm{EC} / \mathrm{DEC}_{(3 / 7)}\end{array}$ & $\begin{array}{l}\text { Initial: } 110 \mathrm{~mA} \cdot \mathrm{h} \cdot \mathrm{g}^{-1} \\
87 \% \text { of the initial capacity was } \\
\text { obtained after } 25 \text { cycles }\end{array}$ & 7 \\
\hline $\begin{array}{l}\text { PTMA:Super P:PVDF } \\
=40: 50: 10 \mathrm{wt} \%\end{array}$ & $\begin{array}{l}\mathrm{P}(\mathrm{VdF}-\mathrm{HFP}) \\
\text { membrane swollen } \\
\text { with } 1 \mathrm{M} \mathrm{LiPF} \text { in } \\
\text { EC/DMC }(1 / 1) \\
\end{array}$ & $\begin{array}{l}\text { Initial: } 111 \mathrm{~mA} \cdot \mathrm{h} \cdot \mathrm{g}^{-1} \\
91 \% \text { of the initial capacity was } \\
\text { obtained after } 100 \text { cycles }\end{array}$ & 8 \\
\hline $\begin{array}{l}\text { PTMA:Super P:PVDF } \\
=40: 50: 10 \mathrm{wt} \%\end{array}$ & $\begin{array}{l}1 \mathrm{MLiPF}_{6} \text { in } \\
\mathrm{EC} / \mathrm{DMC}(1 / 1)\end{array}$ & $\begin{array}{l}\text { Initial: } 111 \mathrm{~mA} \cdot \mathrm{h} \cdot \mathrm{g}^{-1} \\
\sim 90 \% \text { of the initial capacity was } \\
\text { obtained after } 100 \text { cycles }\end{array}$ & 9 \\
\hline $\begin{array}{l}\text { PTMA:graphite:Carbon } \\
\text { black:PVDF }\end{array}$ & $\begin{array}{l}1 \mathrm{MLiPF}_{6} \text { in } \\
\mathrm{EC} / \mathrm{DMC}(1 / 1)\end{array}$ & $\begin{array}{l}\text { Initial: } 76.7 \mathrm{~mA} \cdot \mathrm{h} \cdot \mathrm{g}^{-1} \\
\text { "a rapid fading of the capacity is } \\
\text { observed" to } 26 \%{ }^{\mathrm{a}} \text { of initial capacity } \\
\text { after } 25 \text { cycles }\end{array}$ & 10 \\
\hline $\begin{array}{l}\text { PTMA:Super P:PVDF } \\
=60: 30: 10 \mathrm{wt} \%\end{array}$ & $\begin{array}{l}1 \mathrm{MLiPF}_{6} \text { in } \\
\mathrm{EC} / \mathrm{DMC}(1 / 1)\end{array}$ & $\begin{array}{l}\text { Initial: } 80 \mathrm{~mA} \cdot \mathrm{h} \cdot \mathrm{g}^{-1} \\
\sim 87 \% \text { of the initial capacity was } \\
\text { obtained after } 200 \text { cycles }\end{array}$ & 11 \\
\hline $\begin{array}{l}\text { PTMA:Super P:PVDF } \\
=40: 50: 10 \mathrm{wt} \%\end{array}$ & $\begin{array}{l}1 \mathrm{MLiPF}_{6} \text { in } \\
\mathrm{EC} / \mathrm{DMC}_{(1 / 1)}\end{array}$ & $\begin{array}{l}\text { Initial: } 110 \mathrm{~mA} \cdot \mathrm{h} \cdot \mathrm{g}^{-1} \\
96 \% \text { of the initial capacity was } \\
\text { obtained after } 400 \text { cycles }\end{array}$ & 12 \\
\hline PTMA:Super $\mathrm{P}=25: 75 \mathrm{wt} \%$ & $\begin{array}{l}1 \mathrm{M} \mathrm{LiClO}_{4} \text { in } \\
\mathrm{EC} / \mathrm{DEC}(1 / 1)\end{array}$ & $\begin{array}{l}\text { Initial: } 100^{\mathrm{a}} \mathrm{mA} \cdot \mathrm{h} \cdot \mathrm{g}^{-1} \\
82 \% \text { of the initial capacity was } \\
\text { obtained after } 300 \text { cycles (PTMA } \\
\text { brushes were also fabricated to } \\
\text { prevent dissolution) }\end{array}$ & 13 \\
\hline
\end{tabular}




\begin{tabular}{|c|c|c|c|}
\hline $\begin{array}{l}\text { PTMA:carbon } \\
\text { black:graphene:PVDF } \\
=10: 20: 60: 10 \%\end{array}$ & $\begin{array}{l}1 \mathrm{M} \mathrm{LiPF}_{6} \text { in } \\
\mathrm{EC} / \mathrm{DEC}_{\mathrm{DMC}} \\
(1 / 1 / 1)\end{array}$ & $\begin{array}{l}\text { Initial: } 120^{\mathrm{b}} \mathrm{mA} \cdot \mathrm{h} \cdot \mathrm{g}^{-1} \\
63 \% \text { of the initial capacity was } \\
\text { obtained after } 100 \text { cycles }\end{array}$ & 14 \\
\hline $\begin{array}{l}\text { PTMA composite:Super } \\
\text { P:PVDF } \\
=80: 10: 10 \mathrm{wt} \%\end{array}$ & $\begin{array}{l}1 \mathrm{MLiPF}_{6} \text { in } \\
\mathrm{EC} / \mathrm{DMC}_{(1 / 1)}\end{array}$ & $\begin{array}{l}\text { Initial: } 110 \mathrm{~mA} \cdot \mathrm{h} \cdot \mathrm{g}^{-1} \\
90 \% \text { of the initial capacity was } \\
\text { obtained after } 50 \text { cycles }\end{array}$ & 15 \\
\hline PTMA brushes on ITO glass & $\begin{array}{l}1 \mathrm{M} \text { LiTFSI in } \\
\mathrm{EC} / \mathrm{DEC}(1 / 1)\end{array}$ & $\begin{array}{l}\text { Initial: } 94 \mathrm{~mA} \cdot \mathrm{h} \cdot \mathrm{g}^{-1} \\
97 \% \text { of the initial capacity was } \\
\text { obtained after } 100 \text { cycles (brushes to } \\
\text { prevent dissolution) }\end{array}$ & 16 \\
\hline $\begin{array}{l}\text { PTMA:Super P:PVDF = } \\
25: 65: 10 \mathrm{wt} \%\end{array}$ & $1 \mathrm{M} \mathrm{LiPF}_{6}$ in $\mathrm{DMC}$ & $\begin{array}{l}\text { Initial: } 30 \text { to } 67 \mathrm{~mA} \cdot \mathrm{h} \cdot \mathrm{g}^{-1} \\
0.017 \% \text { to } 0.11 \% \text { capacity loss per } \\
\text { cycle }\end{array}$ & 17 \\
\hline PTMA & $\begin{array}{l}0.5 \mathrm{M} \mathrm{LiCF}_{3} \mathrm{SO}_{3} \text { in } \\
\mathrm{PC}\end{array}$ & $\begin{array}{l}14 \mathrm{wt} \% \text { mass loss over } 200 \mathrm{~s} \text { during } \\
\text { cyclic voltammetry }\end{array}$ & 18 \\
\hline $\begin{array}{l}\text { PTMA } \\
\text { copolymer:VGCF:PVDF = } \\
\text { 30:60:10 wt } \%\end{array}$ & $\begin{array}{l}1 \mathrm{M} \mathrm{LiPF}_{6} \text { in } \\
\mathrm{EC} / \mathrm{DMC}(1 / 1)\end{array}$ & $\begin{array}{l}\text { Initial: } 75^{\mathrm{a}} \mathrm{mA} \cdot \mathrm{h} \cdot \mathrm{g}^{-1} \\
86 \% \text { to } 94 \% \text { of the initial capacity was } \\
\text { retained after } 50 \text { cycles (crosslinked } \\
\text { PTMA was also demonstrated to } \\
\text { prevent dissolution) }\end{array}$ & 19 \\
\hline $\begin{array}{l}\text { PTMA: Super P:PVDF = } \\
\text { 10:80:10 wt } \%\end{array}$ & $\begin{array}{l}1 \mathrm{M} \mathrm{LiPF}_{6} \text { in } \\
\operatorname{EC} / \mathrm{DMC}(1 / 1)\end{array}$ & $\begin{array}{l}\text { Initial: } \sim 77.4 \mathrm{~mA} \cdot \mathrm{h} \cdot \mathrm{g}^{-1} \\
\text { "the capacity of PTMA-oxidation } \\
\text { dropped dramatically within the first } \\
30 \text { cycles to } \sim 62 \mathrm{~mA} \cdot \mathrm{h} \cdot \mathrm{g}^{-1} \text { " }\end{array}$ & 20 \\
\hline $\begin{array}{l}\text { PTMA: Super P:PVDF = } \\
\text { 30:60:10 wt } \%\end{array}$ & $\begin{array}{l}1 \mathrm{M} \mathrm{LiPF}_{6} \text { in } \\
\mathrm{EC} / \mathrm{DMC}(1 / 1)\end{array}$ & $\begin{array}{l}\text { Initial: } 28.5 \mathrm{~mA} \cdot \mathrm{h} \cdot \mathrm{g}^{-1} \\
92 \% \text { of the initial capacity was } \\
\text { retained after } 35 \text { cycles }\end{array}$ & This Work \\
\hline $\begin{array}{l}\text { PTMA: Super P:PMMA = } \\
\text { 30:60:10 wt } \%\end{array}$ & $\begin{array}{l}1 \mathrm{M} \mathrm{LiPF}_{6} \text { in } \\
\mathrm{EC}_{\mathrm{DMC}}(1 / 1)\end{array}$ & $\begin{array}{l}\text { Initial: } 54.9 \mathrm{~mA} \cdot \mathrm{h} \cdot \mathrm{g}^{-1} \\
89 \% \text { of the initial capacity was } \\
\text { retained after } 35 \text { cycles }\end{array}$ & This Work \\
\hline
\end{tabular}

EC: ethylene carbonate

DEC: diethyl carbonate

VGCF: vapor-grown carbon fiber

PVDF: poly(vinylidene difluoride)

CMC: carboxymethyl cellulose

PTFE: poly(tetrafluoroethylene)

TBA: tetrabutylammonium

DCM: dichloromethane

ACN: acetonitrile

PC: propylene carbonate

DMC: dimethyl carbonate

${ }^{\text {a}}$ Estimated from plot

${ }^{\mathrm{b}}$ Accessed the three redox states of PTMA with a two-electron process 


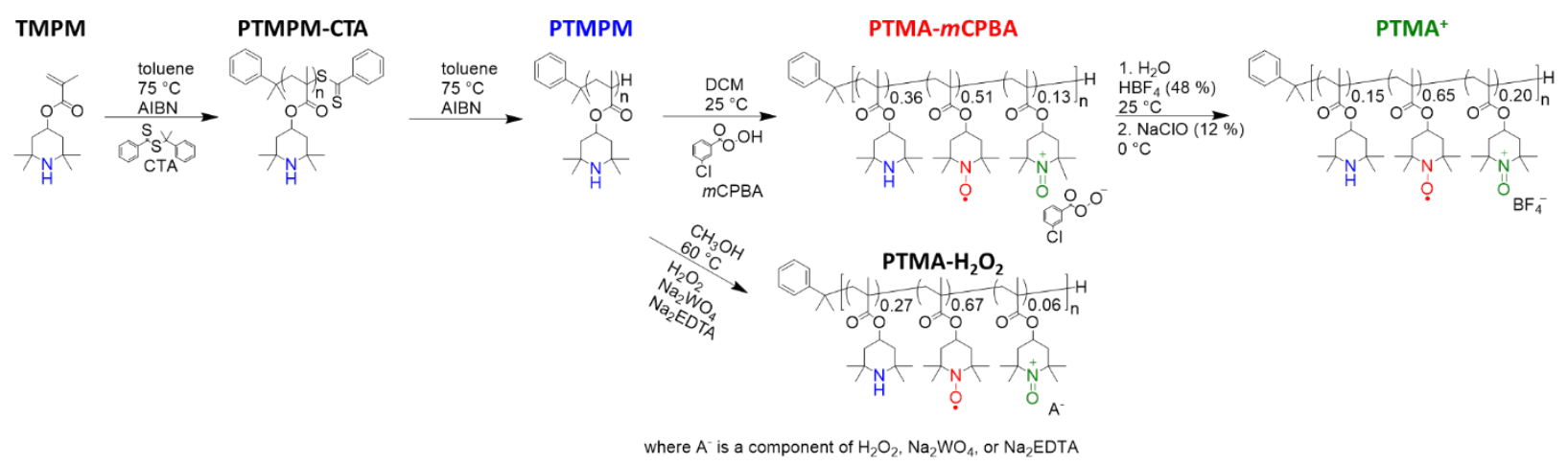

Figure S1. Synthetic route for PTMPM, PTMA- $m$ CPBA, PTMA- $\mathrm{H}_{2} \mathrm{O}_{2}$, and $\mathrm{PTMA}^{+}$.

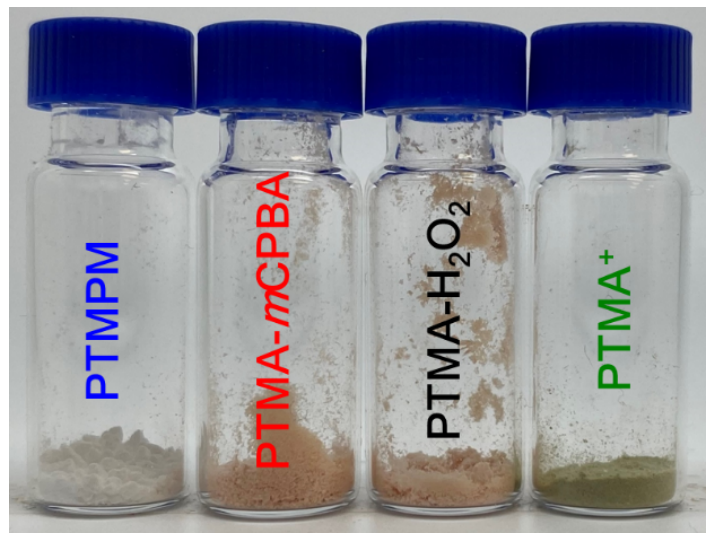

Figure S2. PTMPM, PTMA- $m$ CPBA, PTMA- $\mathrm{H}_{2} \mathrm{O}_{2}$, and $\mathrm{PTMA}^{+}$powders collected after the final vacuum filtration step. 


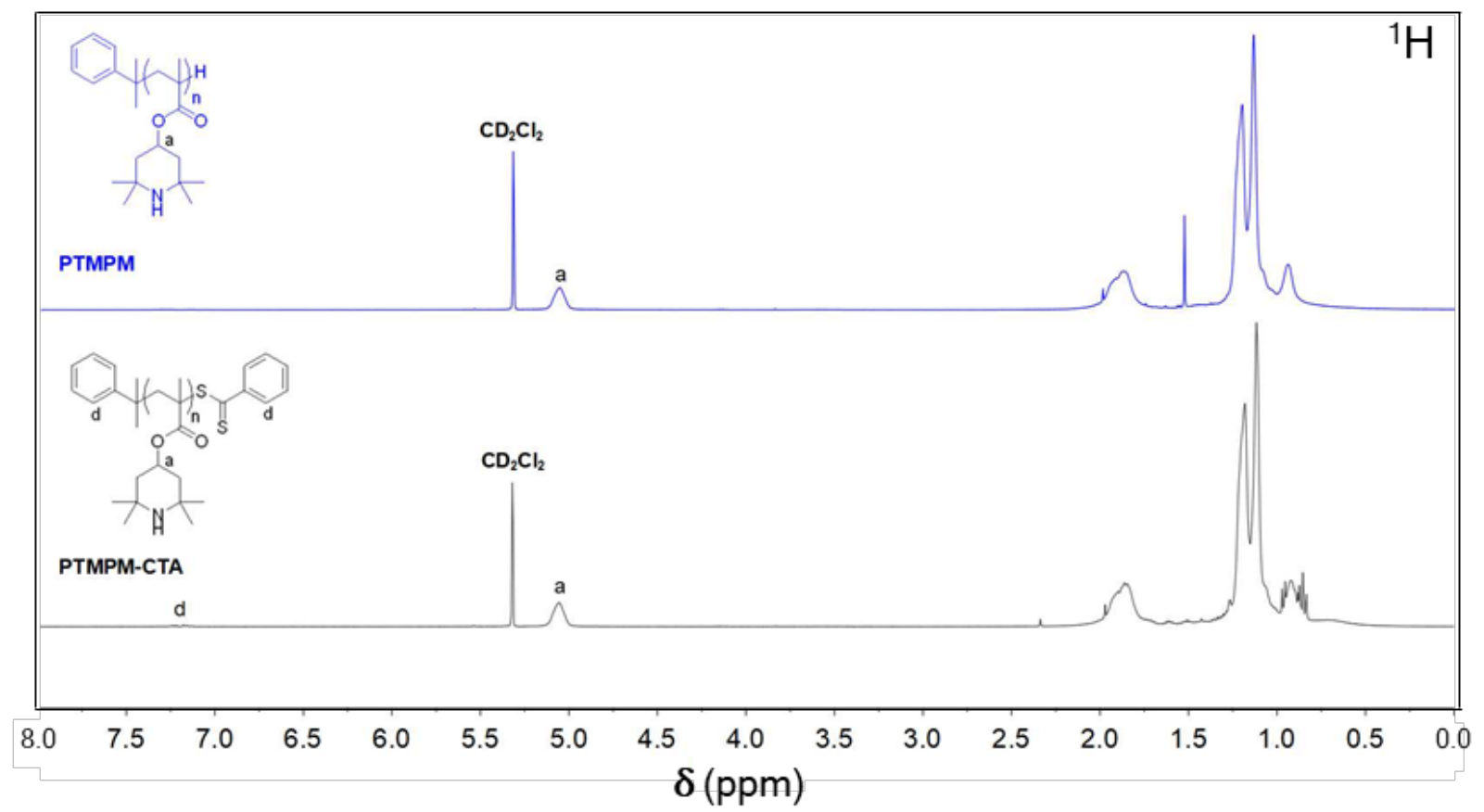

Figure S3. The ${ }^{1} \mathrm{H}-\mathrm{NMR}$ of PTMPM-CTA and PTMPM, showing a loss in the signal between 7.0 and $7.5 \mathrm{ppm}$ to confirm the successful removal of the CTA end group.

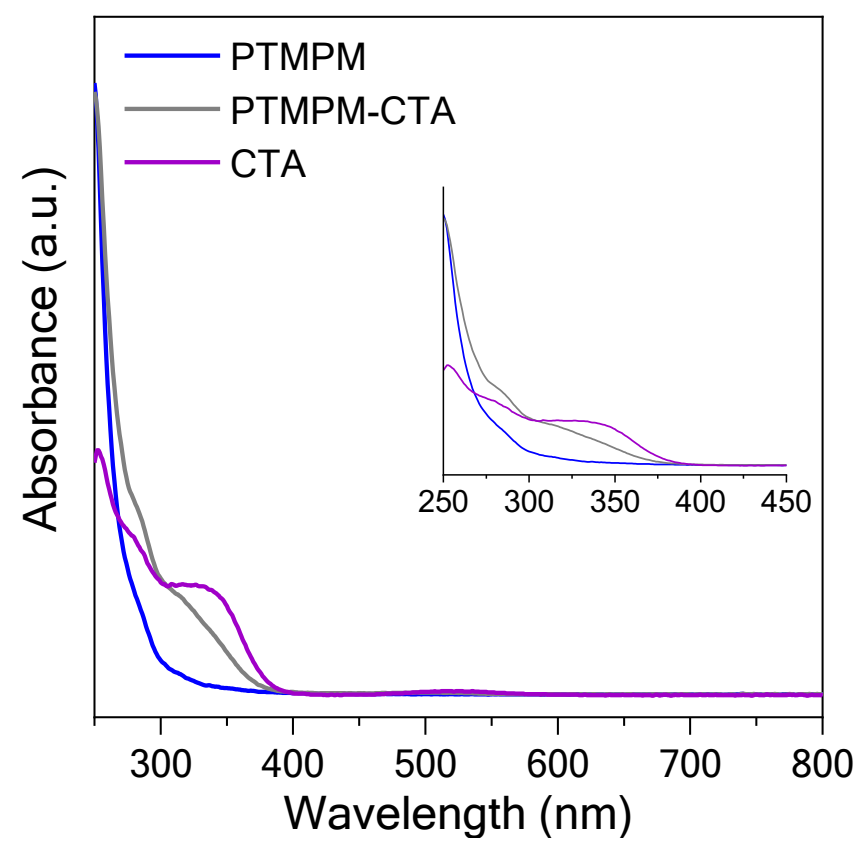

Figure S4. UV-Vis spectra of PTMPM-RAFT and PTMPM, confirming the successful removal of the CTA end group due to the loss of the shoulder between 300 and $400 \mathrm{~nm}$. 
a)

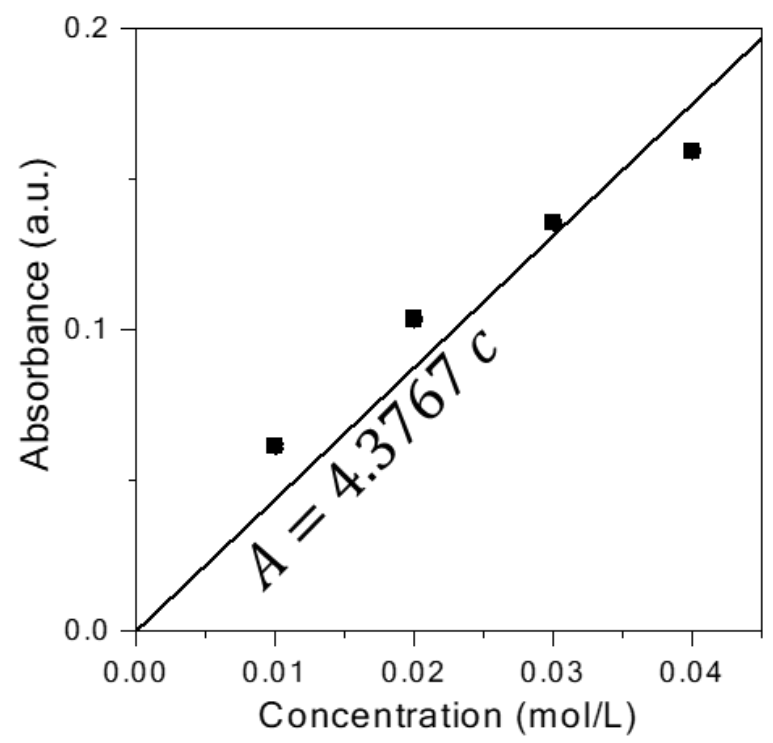

b)

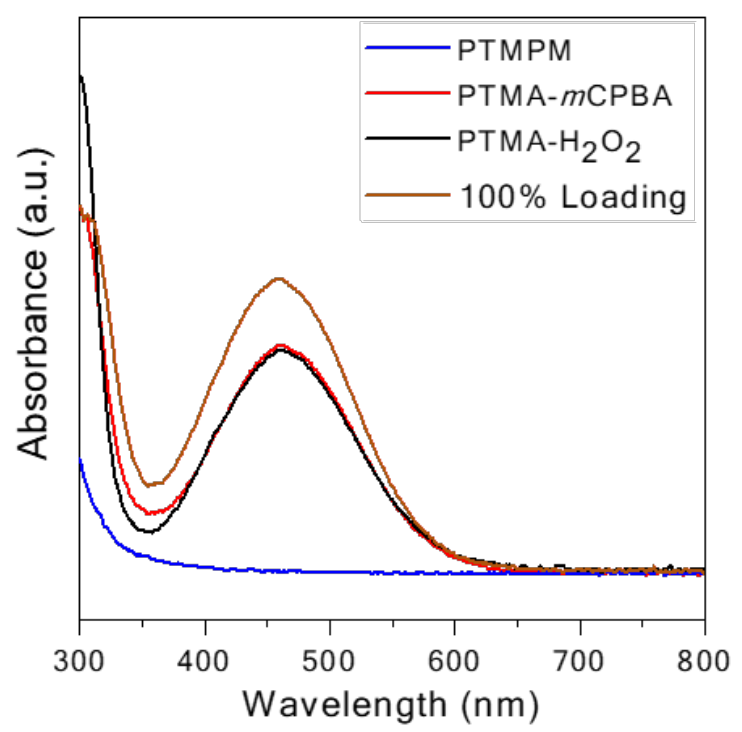

Figure S5. a) The calibration curve for the standard, 4-hydroxy-TEMPO, in chloroform used to determine the radical content of PTMA, and b) the UV-Vis spectra for the standard (or 100\% radical loading) and utilized polymers.

Table S2. Radical loading percentage determined using UV-Vis spectroscopy, EPR spectroscopy, and XPS.

\begin{tabular}{|c|c|c|c|c|}
\hline Method of Measurement & PTMPM & PTMA-mCPBA & PTMA-H $_{2} \mathbf{O}_{2}$ & PTMA $^{+}$ \\
\hline UV-Vis & 0 & 68 & 67 & NA \\
\hline EPR & 0 & 67 & 73 & 13 \\
\hline XPS & 0 & 51 & 67 & 65 \\
\hline Avg. Radical Loading & 0 & $62 \pm 10$ & $69 \pm 3$ & $39 \pm 37$ \\
\hline
\end{tabular}


a)

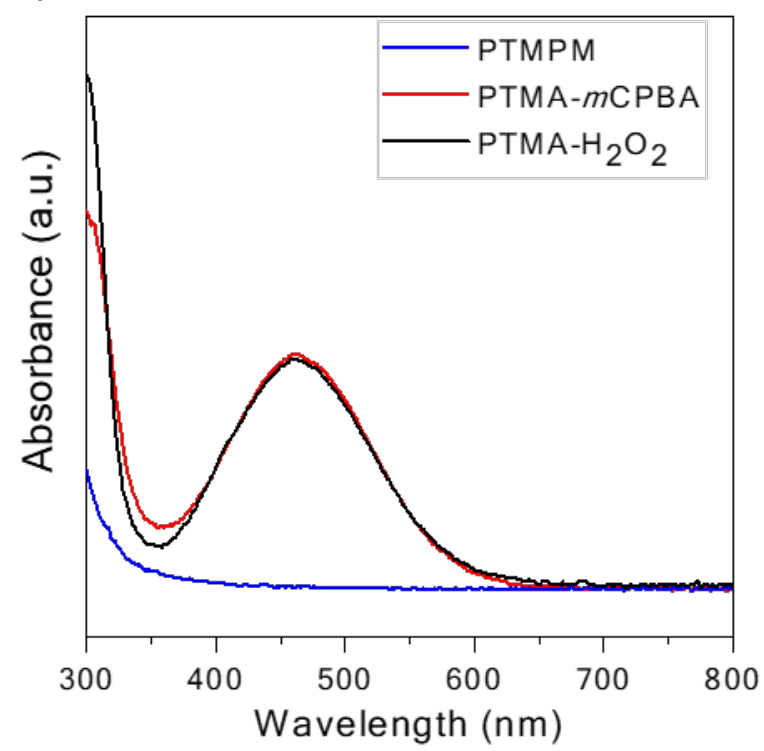

c)

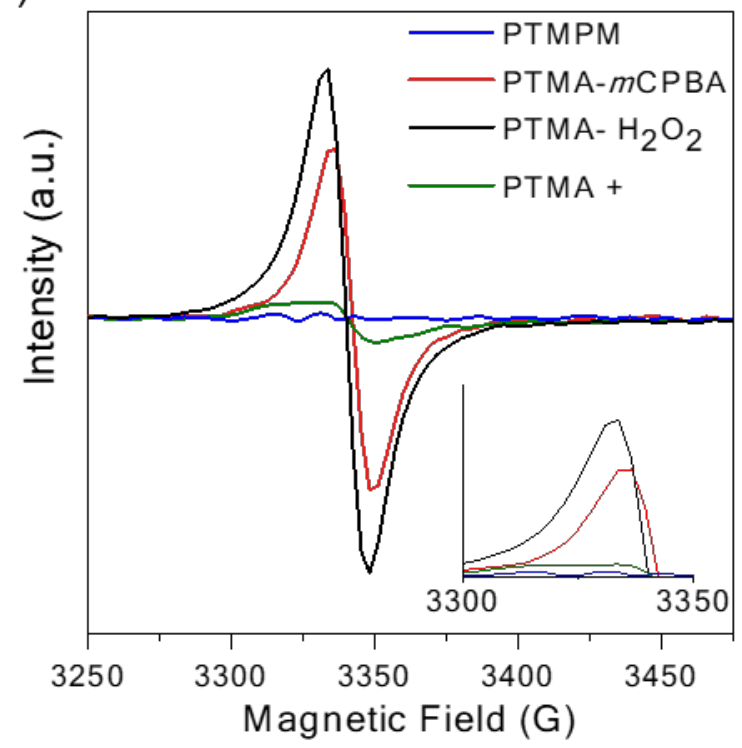

b)

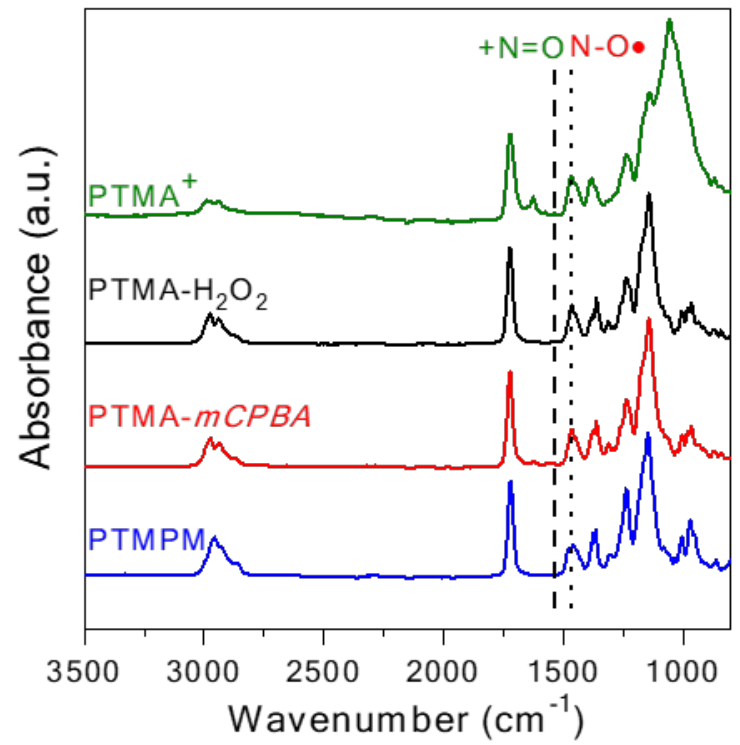

d)

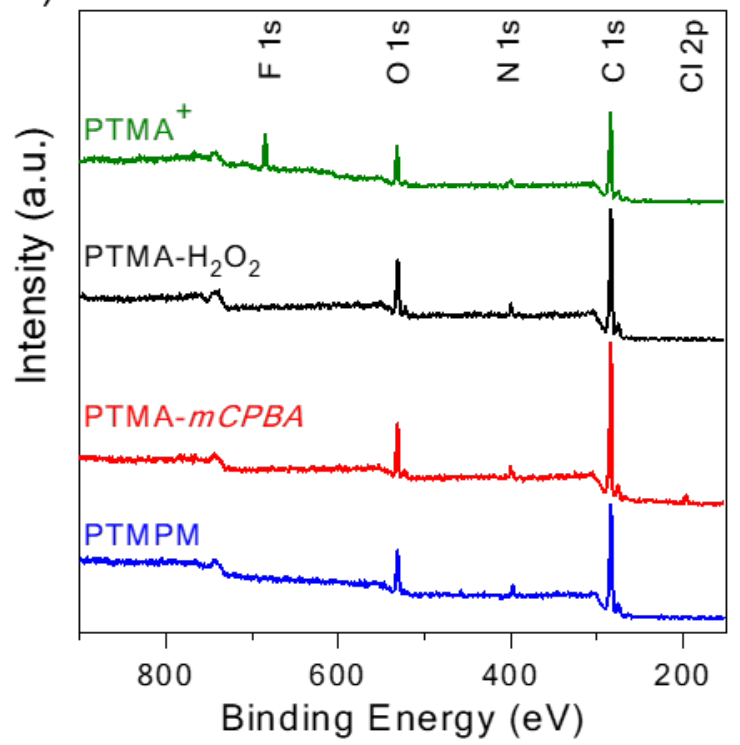

Figure S6. a) UV-Vis spectra, b) FTIR spectra, c) EPR spectra, and d) XPS survey scans for PTMPM, PTMA- $m$ CPBA, PTMA- $\mathrm{H}_{2} \mathrm{O}_{2}$, and $\mathrm{PTMA}^{+}$. $\mathrm{PTMA}^{+} \mathrm{UV}-\mathrm{V}$ is spectra are not shown because PTMA ${ }^{+}$was not soluble in chloroform at the studied concentration. FTIR and XPS spectra were collected for powder samples (except for XPS on PTMA- $m$ CPBA, which was conducted on a thin film on ITO glass). 

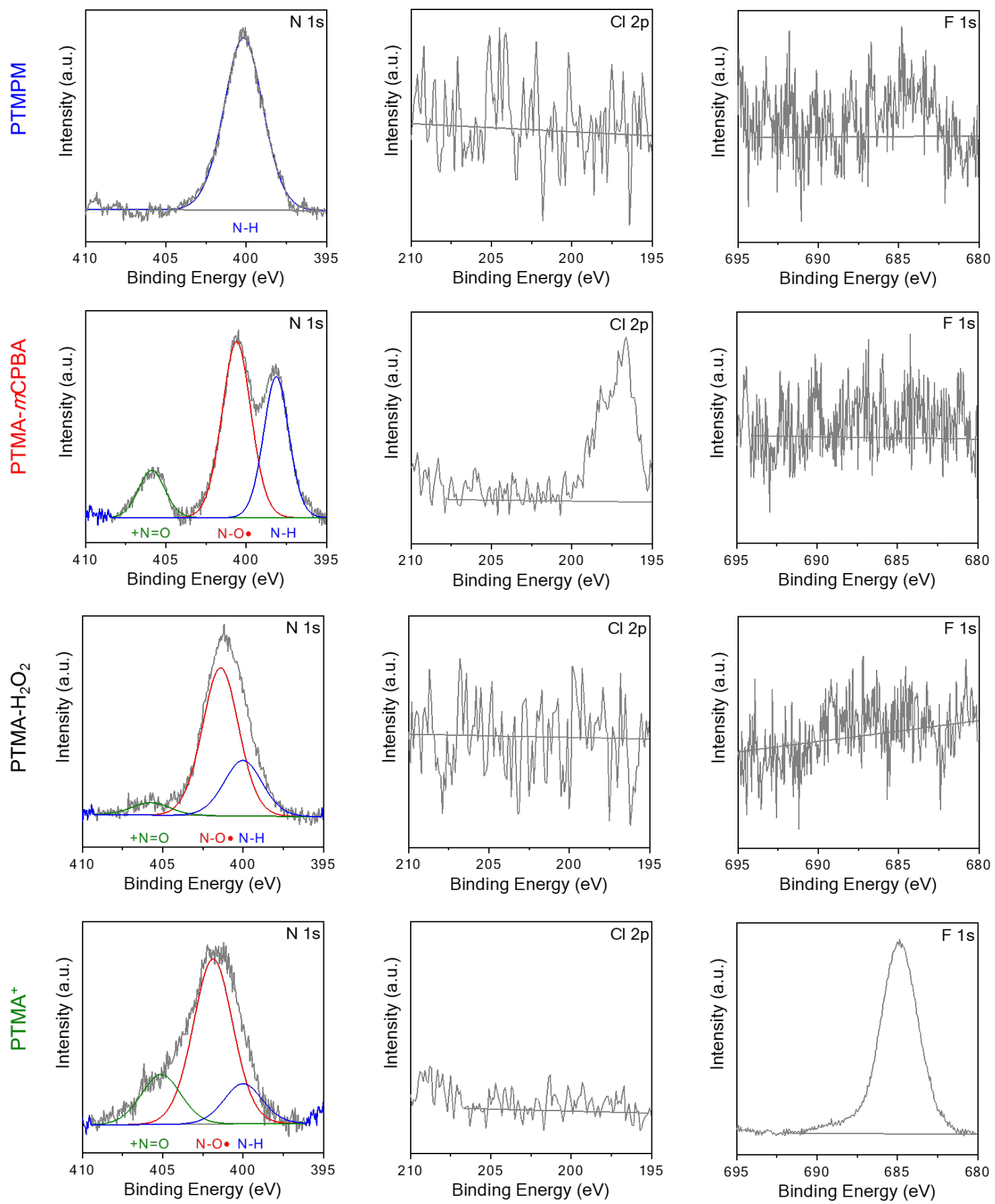

Figure S7. High resolution N 1s, $\mathrm{Cl} 2 \mathrm{p}$, and $\mathrm{F}$ 1s XPS for PTMPM, PTMA- $m$ CPBA, PTMA- $\mathrm{H}_{2} \mathrm{O}_{2}$, and $\mathrm{PTMA}^{+}$. All data was collected from powder samples, except for PTMA- $m$ CPBA, which was a thin film on ITO glass. 
Table S3. Summary of XPS results from deconvolution of the $\mathrm{N}$ 1s peak to determine the functional group distribution of the polymers studied.

\begin{tabular}{|l|l|l|l|l|}
\cline { 2 - 5 } \multicolumn{1}{c|}{} & \multicolumn{1}{c|}{ PTMPM } & PTMA-mCPBA & \multicolumn{1}{c|}{ PTMA-H $_{2} \mathbf{O}_{2}$} & \multicolumn{1}{c|}{ PTMA $^{+}$} \\
\hline C (at \%) & 84 & 83 & 83 & 76 \\
\hline O (at \%) & 15 & 11 & 13 & 13 \\
\hline N (at \%) & 4 & 4 & 4 & 4 \\
\hline $\mathrm{N}-\mathrm{H}(\sim 399.8 \mathrm{eV})$ & $100 \%$ & $36 \%$ & $27 \%$ & $15 \%$ \\
\hline $\mathrm{N}-\mathrm{O} \cdot(\sim 401.3 \mathrm{eV})$ & $0 \%$ & $51 \%$ & $67 \%$ & $65 \%$ \\
\hline$+\mathrm{N}=\mathrm{O}(\sim 405.6 \mathrm{eV})$ & $0 \%$ & $13 \%$ & $6 \%$ & $20 \%$ \\
\hline Cl (at \%) & 0 & 2 & 0 & 0 \\
\hline F (at \%) & 0 & 0 & 0 & 7 \\
\hline
\end{tabular}




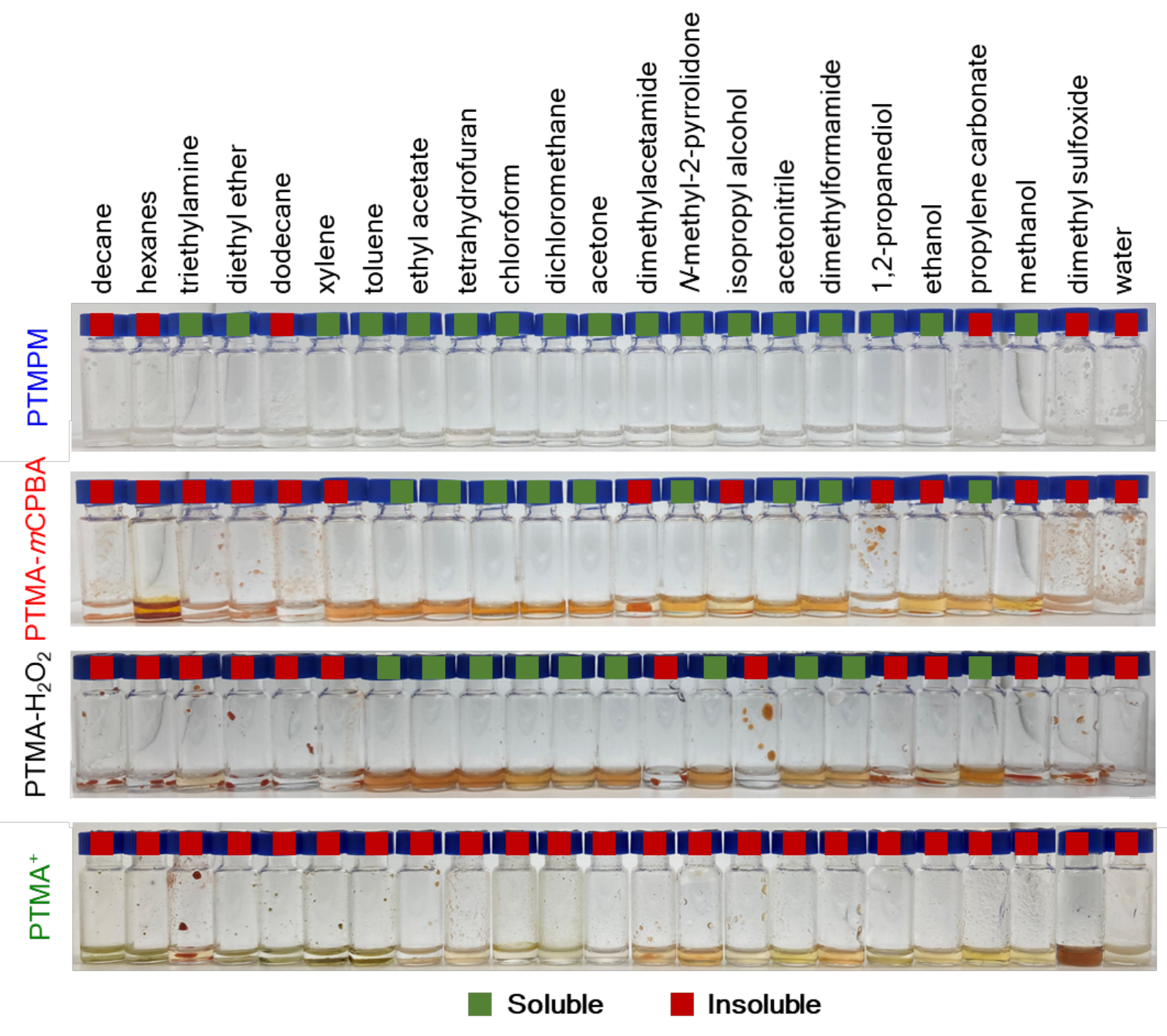

Figure S8. The solubility spectrum for PTMPM, PTMA- $m$ CPBA, PTMA- $\mathrm{H}_{2} \mathrm{O}_{2}$, and PTMA Green indicates the solvents which dissolve the polymer and red indicates those which do not. The Hildebrand solubility parameters vary from 13.5 (decane) to 47.9 (water) $\mathrm{MPa}^{1 / 2}$. 


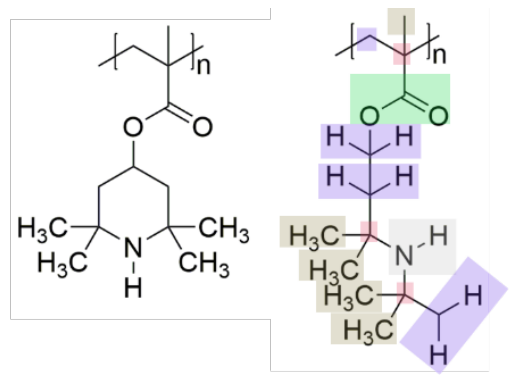

\begin{tabular}{|c|c|c|c|c|c|c|c|c|c|}
\hline \multirow[b]{2}{*}{ Group } & \multirow[b]{2}{*}{$\begin{array}{c}\text { \# in } \\
\text { structure }\end{array}$} & \multicolumn{2}{|c|}{ Hoy } & \multicolumn{2}{|c|}{ Fedors } & \multicolumn{4}{|c|}{ Hansen } \\
\hline & & $\underset{(\mathrm{kJ} / \mathrm{mol})}{\mathrm{U}}$ & $\begin{array}{c}\mathrm{V} \\
\left(\mathrm{cm}^{3} / \mathrm{mol}\right)\end{array}$ & $\frac{F}{\left(\mathrm{~J}^{1 / 2} \mathrm{~cm}^{3 / 2} \mathrm{~mol}^{-1}\right)}$ & $\begin{array}{c}V \\
\left(\mathrm{~cm}^{3} / \mathrm{mol}\right)\end{array}$ & $\begin{array}{c}F_{d} \\
\left(J^{1 / 2} \mathrm{~cm}^{3 / 2} \mathrm{~mol}^{-}\right. \\
1)\end{array}$ & $\begin{array}{c}F_{p} \\
\left(J^{1 / 2} \mathrm{~cm}^{3 / 2} \mathrm{~mol}^{-}\right. \\
1)\end{array}$ & $\begin{array}{c}U_{\mathrm{h}} \\
(\mathrm{J} / \mathrm{mol})\end{array}$ & $\begin{array}{c}\mathrm{V} \\
\left(\mathrm{cm}^{3} / \mathrm{mol}\right)\end{array}$ \\
\hline & 3 & 65.5 & 3.562 & 1.47 & -19.2 & -70 & 0 & 0 & -19.2 \\
\hline & 4 & 269.0 & 15.553 & 4.94 & 16.1 & 270 & 0 & 0 & 16.6 \\
\hline$-\mathrm{CH}_{3}$ & 5 & 303.4 & 21.548 & 4.71 & 33.5 & 419 & 0 & 0 & 31.7 \\
\hline & 1 & 668.1 & 23.728 & 18.00 & 18.0 & 667 & 511 & 7000 & 8.2 \\
\hline & 1 & 368.2 & 11.017 & 8.40 & 4.5 & 160 & 211 & 3100 & 4.5 \\
\hline & 1 & -48.0 & 0 & 1.05 & 16.0 & 190 & 0 & 0 & 13.5 \\
\hline Total & 16 & 3777.8 & 215.383 & 75.17 & 212.8 & 3169 & 1766 & 12000 & 183.8 \\
\hline
\end{tabular}

Figure S9. The Hoy, Fedors, and Hansen group contribution calculation for homopolymer PTMPM. 

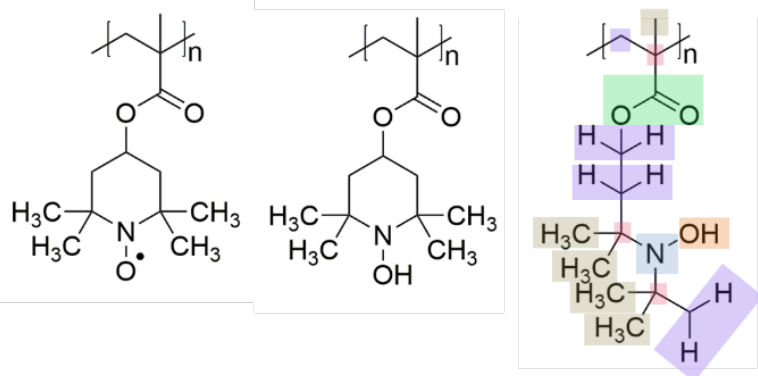

\begin{tabular}{|c|c|c|c|c|c|c|c|c|c|}
\hline \multirow[b]{2}{*}{ Group } & \multirow[b]{2}{*}{$\begin{array}{c}\text { \# in } \\
\text { structure }\end{array}$} & \multicolumn{2}{|c|}{ Hoy } & \multicolumn{2}{|c|}{ Fedors } & \multicolumn{4}{|c|}{ Hansen } \\
\hline & & $\underset{(\mathrm{kJ} / \mathrm{mol})}{U}$ & $\underset{\left(\mathrm{cm}^{3} / \mathrm{mol}\right)}{V}$ & $\frac{F}{\left(J^{1 / 2} \mathrm{~cm}^{3 / 2} \mathrm{~mol}^{-1}\right)}$ & $\underset{\left(\mathrm{cm}^{3} / \mathrm{mol}\right)}{V}$ & $\begin{array}{c}F_{d} \\
\left(\mathrm{~J}^{1 / 2} \mathrm{~cm}^{3 / 2} \mathrm{~mol}^{-}\right. \\
1)\end{array}$ & $\begin{array}{c}F_{p_{1}} \\
\left(\mathrm{~J}^{1 / 2} \mathrm{~cm}^{3 / 2} \mathrm{~mol}^{-}\right. \\
1)\end{array}$ & $\begin{array}{c}U_{h} \\
(\mathrm{~J} / \mathrm{mol})\end{array}$ & $\underset{\left(\mathrm{cm}^{3} / \mathrm{mol}\right)}{V}$ \\
\hline & 3 & 65.5 & 3.562 & 1.47 & -19.2 & -70 & 0 & 0 & -19.2 \\
\hline & 4 & 269.0 & 15.553 & 4.94 & 16.1 & 270 & 0 & 0 & 16.6 \\
\hline$-\mathrm{CH}_{3}$ & 5 & 303.4 & 21.548 & 4.71 & 33.5 & 419 & 0 & 0 & 31.7 \\
\hline & 1 & 668.1 & 23.728 & 18.00 & 18.0 & 667 & 511 & 7000 & 8.2 \\
\hline & 1 & 125.0 & 12.569 & 4.20 & -9.0 & 31 & 149 & 5000 & -9.00 \\
\hline$-\mathrm{OH}$ & 1 & 798.6 & 12.457 & 29.80 & 10.0 & 211 & 499 & 20000 & 10.5 \\
\hline & 1 & -48.0 & 0 & 1.05 & 16.0 & 190 & 0 & 0 & 13.5 \\
\hline Total & 16 & 4333.2 & 229.392 & 100.77 & 209.3 & 4064 & 730 & 32000 & 190.5 \\
\hline
\end{tabular}

Figure S10. The Hoy, Fedors, and Hansen group contribution calculation for homopolymer PTMA. 

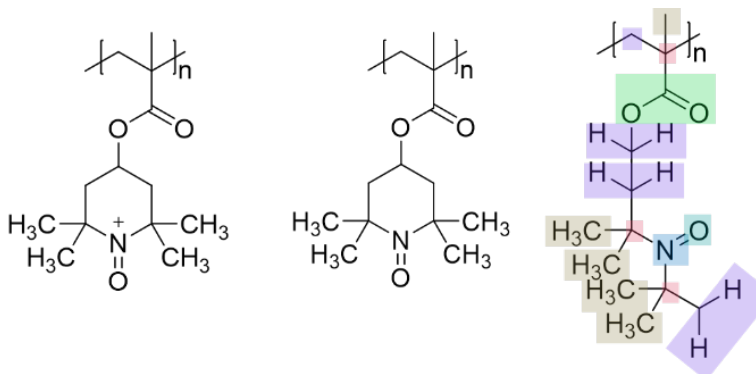

\begin{tabular}{|c|c|c|c|c|c|c|c|c|c|}
\hline \multirow[b]{2}{*}{ Group } & \multirow[b]{2}{*}{$\begin{array}{c}\text { \# in } \\
\text { structure }\end{array}$} & \multicolumn{2}{|c|}{ Hoy } & \multicolumn{2}{|l|}{ Fedors } & \multicolumn{4}{|c|}{ Hansen } \\
\hline & & $\underset{(k J / m o l)}{U}$ & $\begin{array}{c}V \\
\left(\mathrm{~cm}^{3} / \mathrm{mol}\right)\end{array}$ & $\frac{F}{\left(\mathrm{~J}^{1 / 2} \mathrm{~cm}^{3 / 2} \mathrm{~mol}^{-1}\right)}$ & $\underset{\left(\mathrm{cm}^{3} / \mathrm{mol}\right)}{V}$ & $\begin{array}{c}F_{d} \\
\left(\mathrm{~J}^{1 / 2} \mathrm{~cm}^{3 / 2} \mathrm{~mol}^{-}\right. \\
1)\end{array}$ & $\begin{array}{c}\mathrm{F}_{\mathrm{p}} \\
\left(\mathrm{J}^{1 / 2} \mathrm{Cm}^{3 / 2} \mathrm{~mol}^{-}\right. \\
1)\end{array}$ & $\begin{array}{c}U_{h} \\
(\mathrm{~J} / \mathrm{mol})\end{array}$ & $\begin{array}{c}\mathrm{V} \\
\left(\mathrm{cm}^{3} / \mathrm{mol}\right)\end{array}$ \\
\hline & 3 & 65.5 & 3.562 & 1.47 & -19.2 & -70 & 0 & 0 & -19.2 \\
\hline & 4 & 269.0 & 15.553 & 4.94 & 16.1 & 270 & 0 & 0 & 16.6 \\
\hline$-\mathrm{CH}_{3}$ & 5 & 303.4 & 21.548 & 4.71 & 33.5 & 419 & 0 & 0 & 31.7 \\
\hline$-\mathrm{CO}_{2}$ & 1 & 668.1 & 23.728 & 18.00 & 18.0 & 667 & 511 & 7000 & 8.2 \\
\hline & 1 & 125.0 & 12.569 & 4.20 & -9.0 & 164 & 1323 & 5000 & 4.0 \\
\hline & 1 & 235.2 & 6.462 & 3.35 & 3.8 & 100 & 401 & 300 & 3.8 \\
\hline & 1 & -48.0 & 0 & 1.05 & 16.0 & 190 & 0 & 0 & 13.5 \\
\hline Total & 16 & 3769.8 & 223.397 & 74.32 & 203.1 & 4086 & 1473.856 & 15000 & 196.8 \\
\hline
\end{tabular}

Figure S11. The Hoy, Fedors, and Hansen group contribution calculation for homopolymer PTMA $^{+}$. 
Table S4. Group contribution of the studied polymers with adjustment for the different fractions of comonomers in the tri-copolymers (PTMA-mCPBA, PTMA- $\mathrm{H}_{2} \mathrm{O}_{2}$, and PTMA ${ }^{+}$).

\begin{tabular}{|c|c|c|c|c|c|c|c|c|c|c|}
\hline & & & \multicolumn{2}{|c|}{ Ноу } & \multicolumn{2}{|c|}{ Fedors } & \multicolumn{4}{|c|}{ Hansen } \\
\hline & 气̊ํํํ & : & 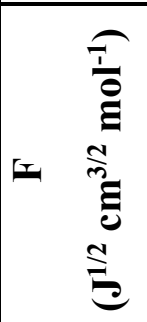 & 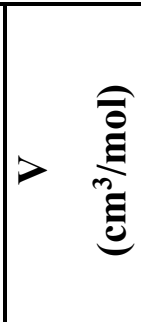 & 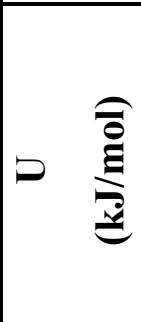 & 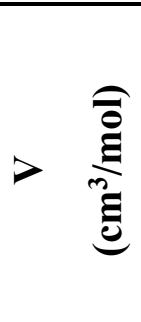 & 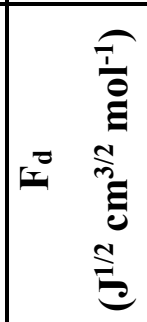 & 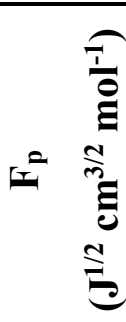 & $=\stackrel{\overparen{\frac{1}{0}}}{\stackrel{\Xi}{\Xi}}$ & 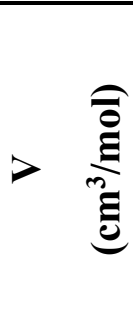 \\
\hline \multirow{4}{*}{$\sum_{i=1}^{\infty}$} & $\mathrm{N}-\mathrm{H}$ & 1.00 & 3777.8 & 215.4 & 75.2 & 212.8 & 3982 & 552.8 & 10100 & 193.5 \\
\hline & $\mathrm{N}-\mathrm{O} \bullet$ & 0 & 0 & 0 & 0 & 0 & 0 & 0 & 0 & 0 \\
\hline & $+\mathrm{N}=\mathrm{O}$ & 0 & 0 & 0 & 0 & 0 & 0 & 0 & 0 & 0 \\
\hline & \multicolumn{2}{|c|}{ Sum } & 3777.8 & 215.4 & 75.2 & 212.8 & 3982 & 552.8 & 10100 & 193.5 \\
\hline \multirow{4}{*}{ 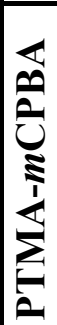 } & N-H & 0.36 & 1360.0 & 77.6 & 27.1 & 76.6 & 1433.5 & 199.0 & 3636 & 69.7 \\
\hline & $\mathrm{N}-\mathrm{O} \bullet$ & 0.51 & 2209.9 & 117.0 & 51.4 & 106.7 & 2072.6 & 372.1 & 16320 & 97.1 \\
\hline & $+\mathrm{N}=\mathrm{O}$ & 0.13 & 490.1 & 29.0 & 9.7 & 26.4 & 531.2 & 191.6 & 1950 & 25.6 \\
\hline & \multicolumn{2}{|c|}{ Sum } & 4060.0 & 223.7 & 88.1 & 209.8 & 4037.3 & 762.7 & 21906 & 192.4 \\
\hline \multirow{4}{*}{ 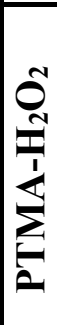 } & $\mathrm{N}-\mathrm{H}$ & 0.27 & 1020.0 & 58.2 & 20.3 & 57.5 & 1075.1 & 149.3 & 2727 & 52.2 \\
\hline & $\mathrm{N}-\mathrm{O} \bullet$ & 0.67 & 2903.2 & 153.7 & 67.5 & 140.2 & 2722.9 & 488.8 & 21440 & 127.6 \\
\hline & $+\mathrm{N}=\mathrm{O}$ & 0.06 & 226.2 & 13.4 & 4.5 & 12.2 & 245.2 & 88.4 & 900 & 11.8 \\
\hline & \multicolumn{2}{|c|}{ Sum } & 4149.4 & 225.2 & 92.3 & 209.9 & 4043.2 & 726.5 & 25067 & 191.7 \\
\hline \multirow{4}{*}{ 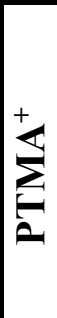 } & $\mathrm{N}-\mathrm{H}$ & 0.15 & 566.7 & 32.3 & 11.3 & 31.9 & 597.3 & 82.9 & 1515 & 29.0 \\
\hline & $\mathrm{N}-\mathrm{O} \bullet$ & 0.65 & 2816.6 & 149.1 & 65.5 & 136.0 & 2641.6 & 474.2 & 20800 & 123.8 \\
\hline & $+\mathrm{N}=\mathrm{O}$ & 0.2 & 754.0 & 44.7 & 14.9 & 40.6 & 817.2 & 294.8 & 3000 & 39.4 \\
\hline & \multicolumn{2}{|c|}{ Sum } & 4137.2 & 226.1 & 91.6 & 208.6 & 4056.1 & 851.9 & 25315 & 192.2 \\
\hline
\end{tabular}


a)

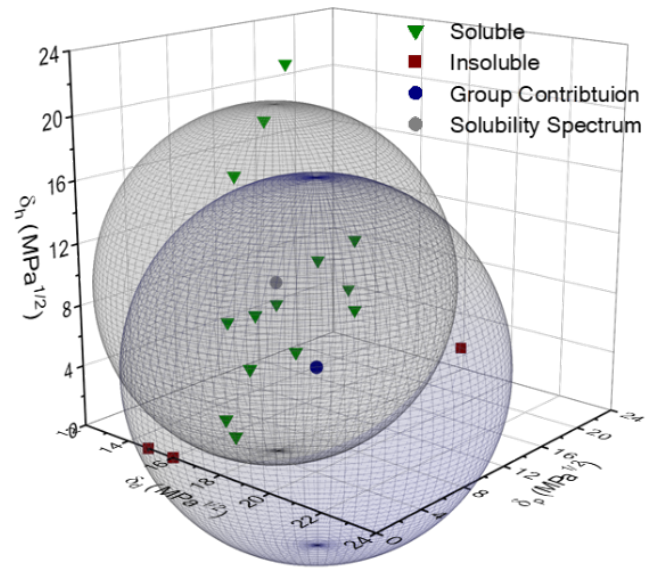

c)

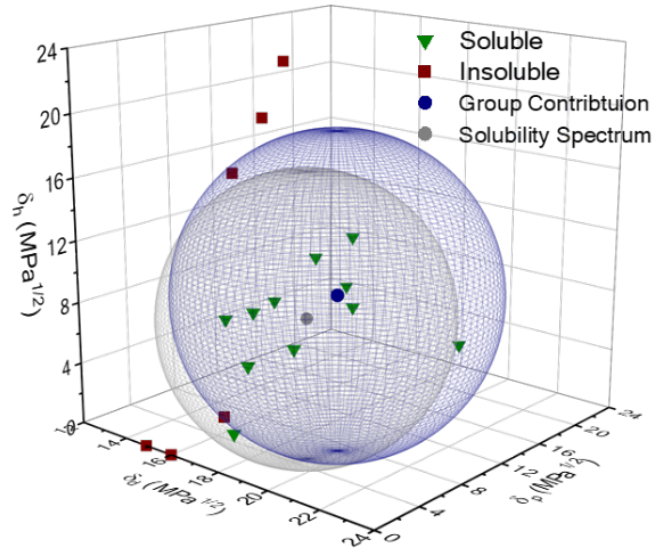

b)

PTMA-mCPBA

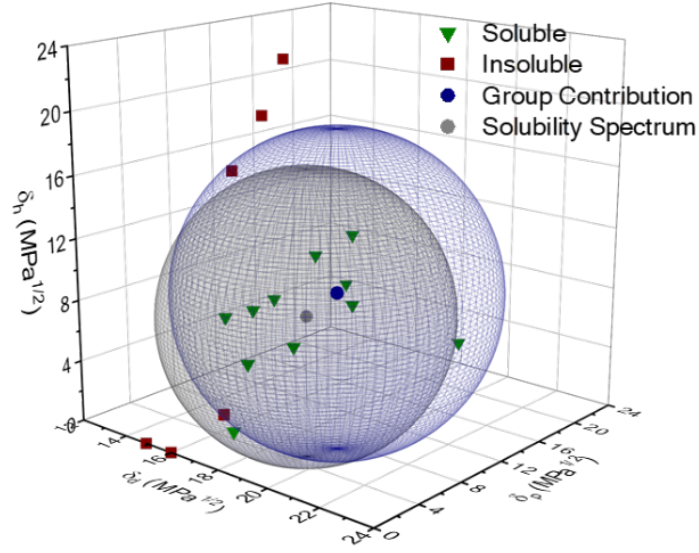

d)

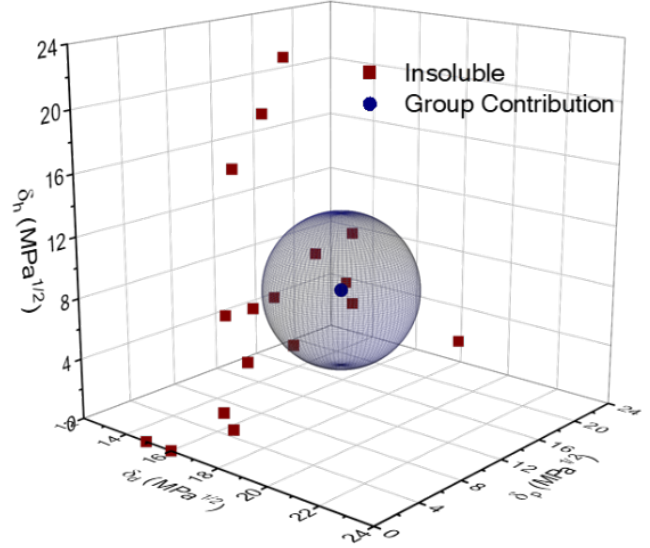

Figure S12. The Hansen solubility spheres for PTMPM, PTMA- $m$ CPBA, PTMA- $\mathrm{H}_{2} \mathrm{O}_{2}$, and PTMA $^{+}$. The 2D projections can be found in Figure 2. Water is off the scale of the graphs due a large $\delta_{n}$ value of $42.4 \mathrm{MPa}^{\prime \prime}$. 

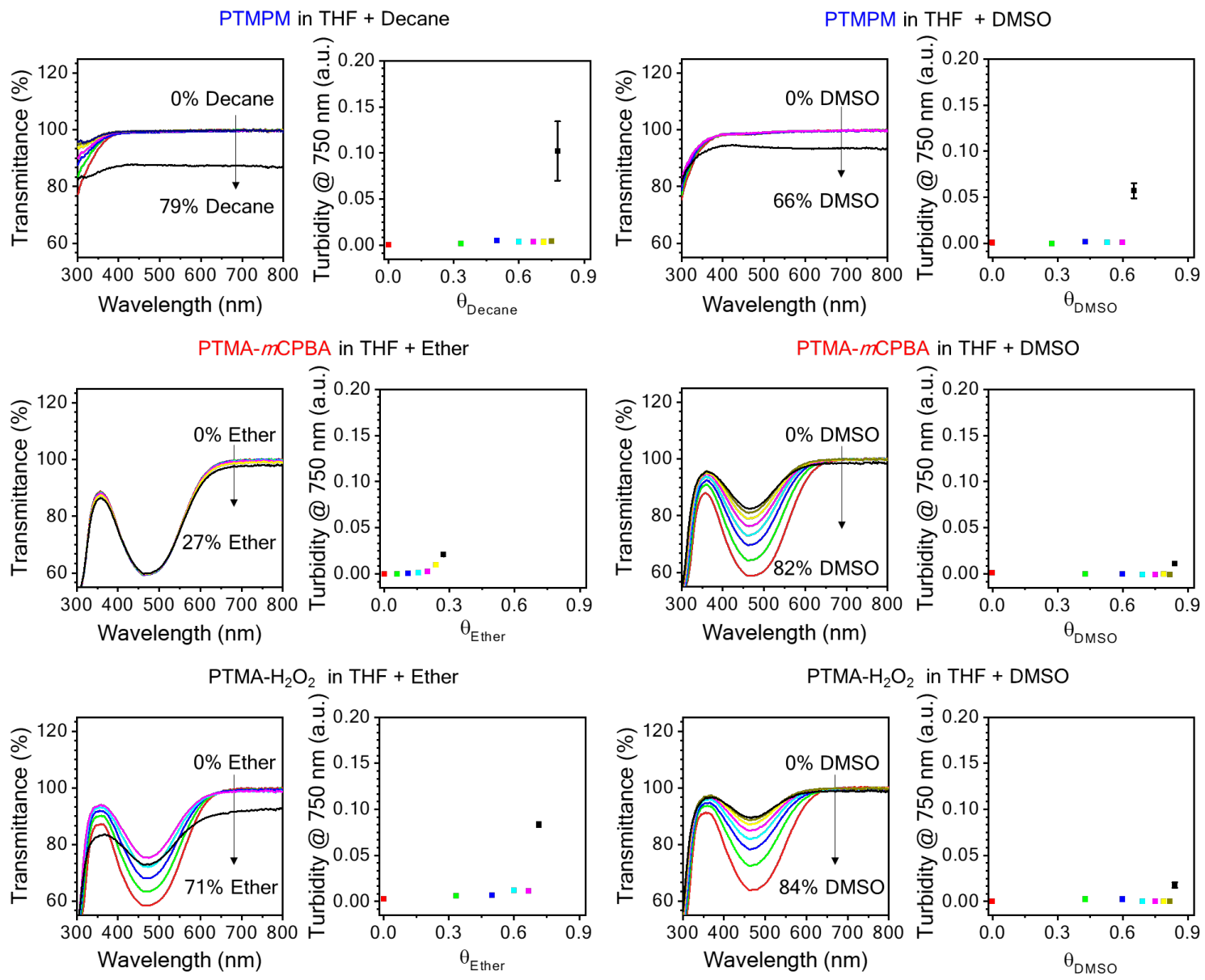

Figure S13. The UV-Vis spectra and corresponding turbidity plots (at $750 \mathrm{~nm}$ ) calculated as a function of the volume fraction of poor solvent added during the titration for polymer solutions in tetrahydrofuran (THF). 

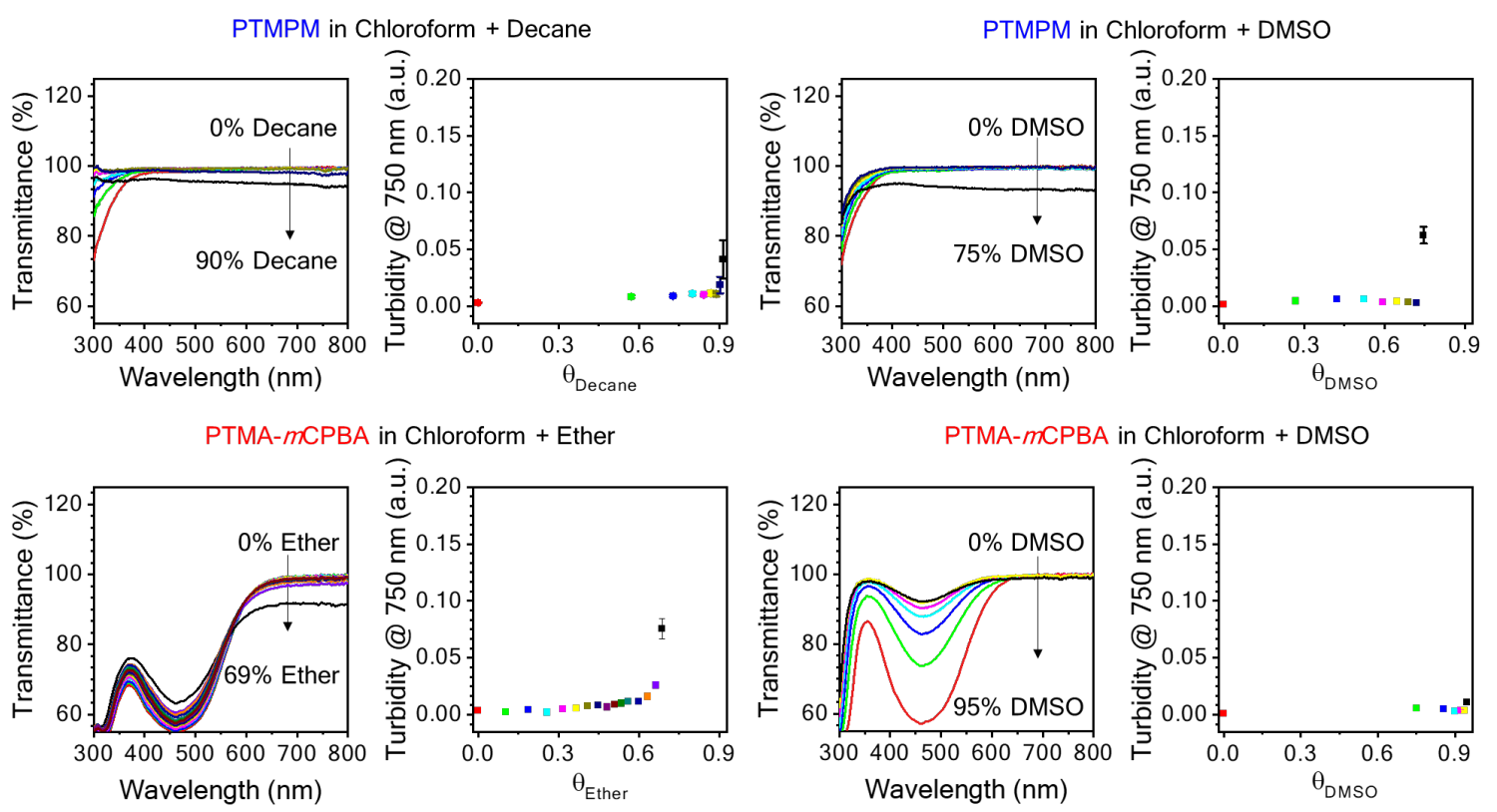

PTMA- $\mathrm{H}_{2} \mathrm{O}_{2}$ in Chloroform + Ether
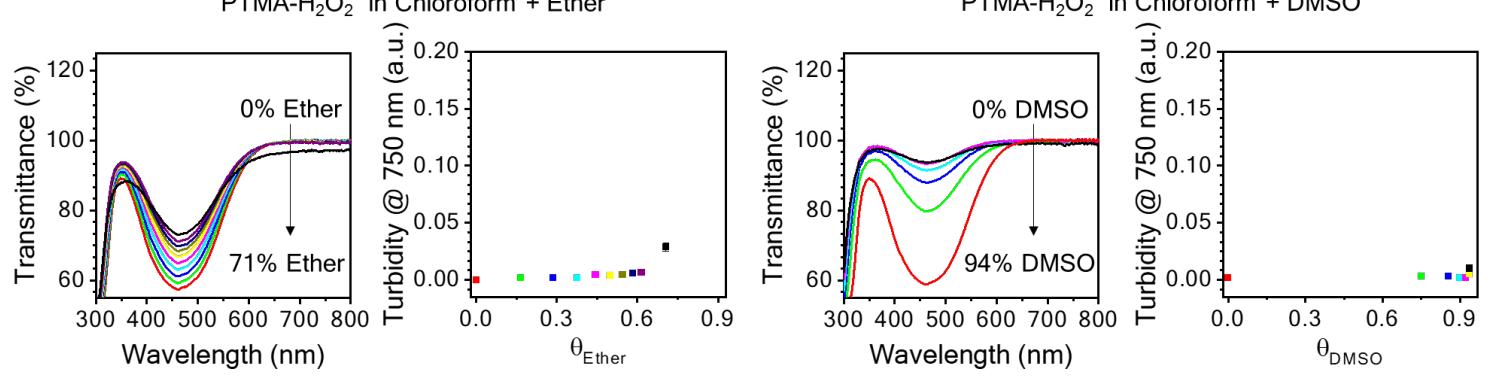

Figure S14. The UV-Vis spectra and corresponding turbidity plots (at $750 \mathrm{~nm}$ ) calculated as a function of the volume fraction of poor solvent added during the titration for polymer solutions in chloroform. 

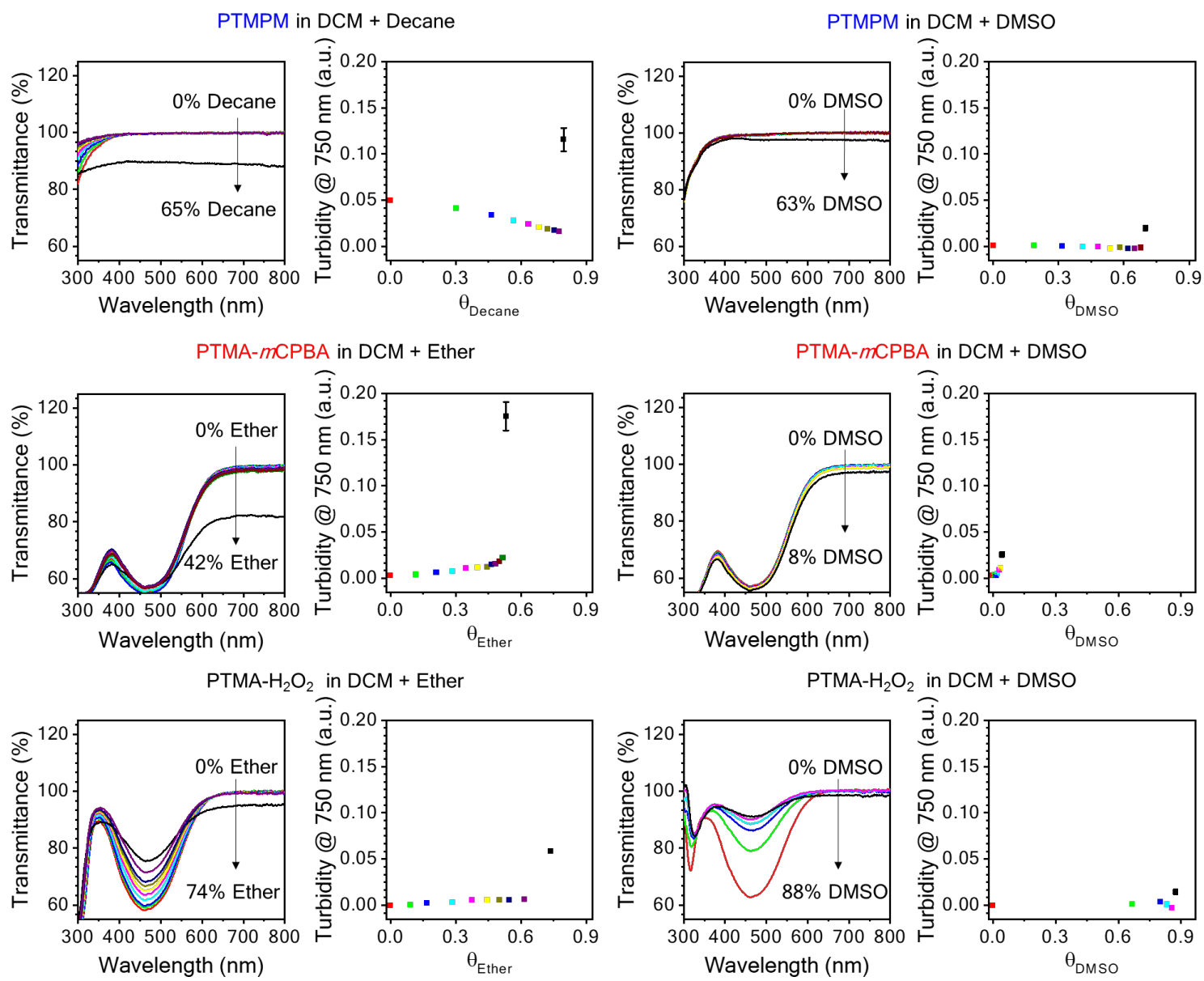

Figure S15. The UV-Vis spectra and corresponding turbidity plots (at $750 \mathrm{~nm}$ ) calculated as a function of the volume fraction of poor solvent added during the titration for polymer solutions in dichloromethane (DCM). 

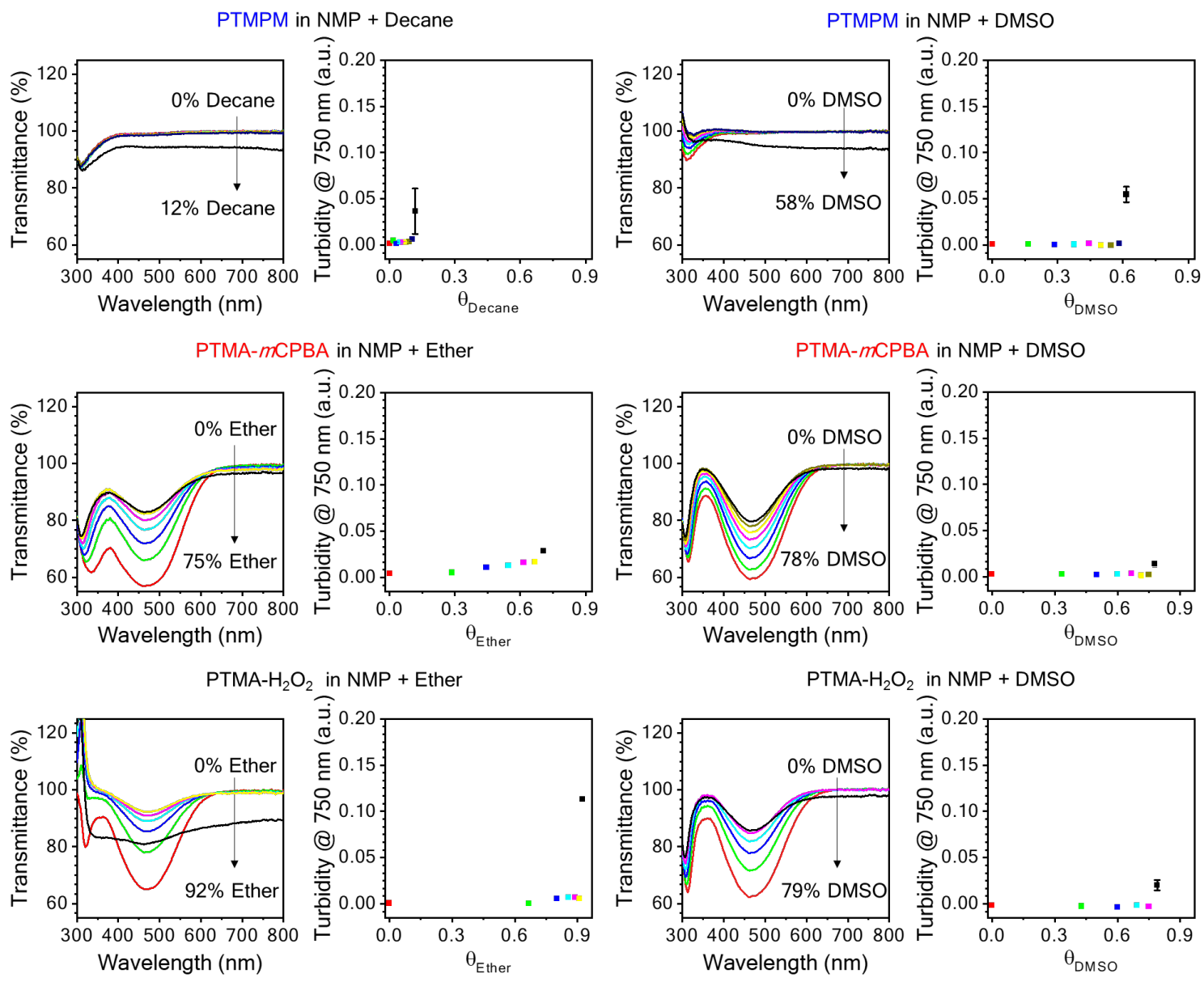

Figure S16. The UV-Vis spectra and corresponding turbidity plots (at $750 \mathrm{~nm}$ ) calculated as a function of the volume fraction of poor solvent added during the titration for polymer solutions in $N$-methyl-2-pyrrolidinone (NMP). 

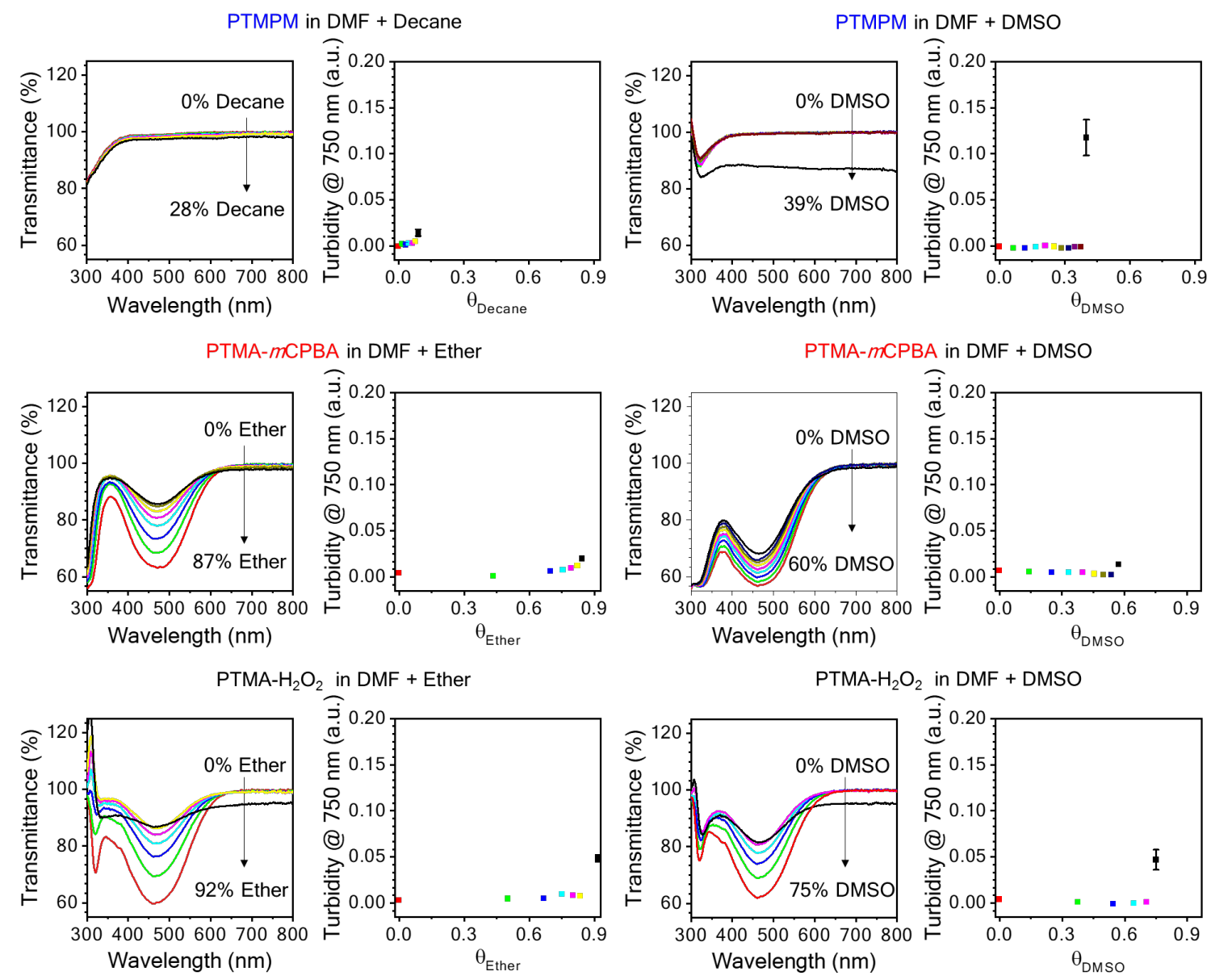

Figure S17. The UV-Vis spectra and corresponding turbidity plots (at $750 \mathrm{~nm}$ ) calculated as a function of the volume fraction of poor solvent added during the titration for polymer solutions in dimethylformamide (DMF).

Table S5. A summary of the turbidity titration results for PTMPM.

\begin{tabular}{|c|c|c|c|c|c|}
\hline \multirow[t]{2}{*}{ Solvent } & \multirow[t]{2}{*}{$\delta_{1}\left(\mathrm{MPa}^{1 / 2}\right)$} & \multirow[t]{2}{*}{$\mathrm{V}\left(\mathrm{cm}^{3} \cdot \mathrm{mol}^{-1}\right)$} & \multicolumn{2}{|c|}{$\Phi$} & \multirow[t]{2}{*}{$\delta_{2}^{\prime}\left(\mathrm{MPa}^{1 / 2}\right)$} \\
\hline & & & Decane & DMSO & \\
\hline THF & 18.6 & 81.7 & $0.79 \pm 0.01$ & $0.66 \pm 0.04$ & $19.2 \pm 0.74$ \\
\hline Chloroform & 19.0 & 80.7 & $0.90 \pm 0.01$ & $0.75 \pm 0.02$ & $19.2 \pm 0.37$ \\
\hline DCM & 19.8 & 63.9 & $0.65 \pm 0.04$ & $0.63 \pm 0.05$ & $20.2 \pm 1.25$ \\
\hline Acetone & 20.3 & 74.0 & $0.57 \pm 0.04$ & $0.60 \pm 0.03$ & $20.7 \pm 0.86$ \\
\hline NMP & 23.1 & 96.5 & $0.12 \pm 0.01$ & $0.58 \pm 0.04$ & $24.3 \pm 0.93$ \\
\hline DMF & 24.8 & 77.0 & $0.20 \pm 0.08$ & $0.39 \pm 0.02$ & $24.5 \pm 1.39$ \\
\hline
\end{tabular}


Table S6. A summary of the turbidity titration results for PTMA- $m$ CPBA.

\begin{tabular}{|c|c|c|c|c|c|}
\hline \multirow[t]{2}{*}{ Solvent } & \multirow[t]{2}{*}{$\delta_{1}\left(\mathrm{MPa}^{1 / 2}\right)$} & \multirow[t]{2}{*}{$\mathrm{V}\left(\mathrm{cm}^{3} \cdot \mathrm{mol}^{-1}\right)$} & \multicolumn{2}{|c|}{$\Phi$} & \multirow[t]{2}{*}{$\delta_{2}^{\prime}\left(\mathrm{MPa}^{1 / 2}\right)$} \\
\hline & & & Ether & DMSO & \\
\hline THF & 18.6 & 81.7 & $0.27 \pm 0.01$ & $0.82 \pm 0.02$ & $22.5 \pm 0.44$ \\
\hline Chloroform & 19.0 & 80.7 & $0.69 \pm 0.01$ & $0.95 \pm 0.01$ & $22.3 \pm 0.17$ \\
\hline DCM & 19.8 & 63.9 & $0.50 \pm 0.03$ & $0.08 \pm 0.07$ & $18.9 \pm 1.47$ \\
\hline Acetone & 20.3 & 74.0 & $0.59 \pm 0.02$ & $0.82 \pm 0.05$ & $22.3 \pm 1.10$ \\
\hline NMP & 23.1 & 96.5 & $0.75 \pm 0.05$ & $0.78 \pm 0.01$ & $22.2 \pm 0.87$ \\
\hline DMF & 24.8 & 77.0 & $0.87 \pm 0.02$ & $0.60 \pm 0.08$ & $21.6 \pm 1.75$ \\
\hline
\end{tabular}

Table S7. A summary of the turbidity titration results for PTMA- $\mathrm{H}_{2} \mathrm{O}_{2}$.

\begin{tabular}{|c|c|c|c|c|c|}
\hline \multirow[t]{2}{*}{ Solvent } & \multirow[t]{2}{*}{$\delta_{1}\left(\mathrm{MPa}^{1 / 2}\right)$} & \multirow[t]{2}{*}{$\mathrm{V}\left(\mathrm{cm}^{3} \cdot \mathrm{mol}^{-1}\right)$} & \multicolumn{2}{|c|}{$\Phi$} & \multirow[t]{2}{*}{$\delta_{2}^{\prime}\left(\mathrm{MPa}^{1 / 2}\right)$} \\
\hline & & & Ether & DMSO & \\
\hline THF & 18.6 & 81.7 & $0.75 \pm 0.03$ & $0.81 \pm 0.03$ & $21.4 \pm 0.82$ \\
\hline Chloroform & 19.0 & 80.7 & $0.72 \pm 0.01$ & $0.95 \pm 0.01$ & $22.2 \pm 0.27$ \\
\hline DCM & 19.8 & 63.9 & $0.75 \pm 0.01$ & $0.89 \pm 0.02$ & $22.1 \pm 0.55$ \\
\hline Acetone & 20.3 & 74.0 & $0.83 \pm 0.01$ & $0.80 \pm 0.01$ & $21.5 \pm 0.10$ \\
\hline NMP & 23.1 & 96.5 & $0.91 \pm 0.02$ & $0.78 \pm 0.01$ & $21.5 \pm 0.43$ \\
\hline DMF & 24.8 & 77.0 & $0.92 \pm 0.02$ & $0.70 \pm 0.04$ & $21.6 \pm 0.88$ \\
\hline
\end{tabular}


For mixed solvent of acetone (dissolving solvent) and ether (non-dissolving solvent):

$$
\begin{gathered}
V_{n d s}=V_{\text {ether }}=89.4 \mathrm{~cm}^{3} / \mathrm{mol} \\
\delta_{n d s}=\delta_{\text {ether }}=15.1 \mathrm{MPa}^{1 / 2} \\
\Phi_{\text {nds }}=\Phi_{\text {ether }}=0.59 \\
V_{d s}=V_{\text {acetone }}=74.0 \mathrm{~cm}^{3} / \mathrm{mol} \\
\delta_{d s}=\delta_{\text {acetone }}=20.3 \mathrm{MPa} a^{1 / 2} \\
\Phi_{d s}=\Phi_{\text {acetone }}=0.41 \\
\delta_{m l}=\Phi_{d s} \delta_{d s}+\Phi_{n d s} \delta_{n d s}=(0.41)\left(20.3 \mathrm{MPa} a^{\frac{1}{2}}\right)+(0.59)\left(15.1 \mathrm{MPa}^{\frac{1}{2}}\right)=17.2 \mathrm{MPa}^{\frac{1}{2}} \\
V_{m l}=\frac{\left(89.4 \frac{\mathrm{cm}^{3}}{\mathrm{~mol}}\right)\left(74.0 \frac{\mathrm{cm}^{3}}{\mathrm{~mol}}\right)}{\left(\Phi_{n d s} V_{d s}+\Phi_{d s} V_{n d s}\right)}=\frac{V_{n d s} V_{d s}}{\left((0.59)\left(74.0 \frac{\mathrm{cm}^{3}}{\mathrm{~mol}^{3}}\right)+(0.41)\left(89.4 \frac{\mathrm{cm}^{3}}{\mathrm{~mol}^{2}}\right)\right)}=89.4 \mathrm{~cm}^{3} / \mathrm{mol}
\end{gathered}
$$

For mixed solvent of acetone (dissolving solvent) and DMSO (non-dissolving solvent):

$$
\begin{aligned}
& V_{n d s}=V_{D M S O}=71.3 \mathrm{~cm}^{3} / \mathrm{mol} \\
& \delta_{n d s}=\delta_{D M S O}=29.7 \mathrm{MPa}^{1 / 2} \\
& \Phi_{n d s}=\Phi_{D M S O}=0.82 \\
& V_{d s}=V_{\text {acetone }}=74.0 \mathrm{~cm}^{3} / \mathrm{mol} \\
& \delta_{d s}=\delta_{\text {acetone }}=20.3 \mathrm{MPa}^{1 / 2} \\
& \Phi_{d s}=\Phi_{\text {acetone }}=0.18 \\
& \delta_{m h}=\Phi_{d s} \delta_{d s}+\Phi_{d s} \delta_{d s}=(0.18)\left(20.3 M P a^{\frac{1}{2}}\right)+(0.82)\left(29.7 M P a^{\frac{1}{2}}\right)=28.0 M P a^{\frac{1}{2}} \\
& V_{m h}=\frac{V_{n d s} V_{d s}}{\left(\Phi_{n d s} V_{d s}+\Phi_{d s} V_{n d s}\right)}==\frac{\left(71.3 \frac{\mathrm{cm}^{3}}{\mathrm{~mol}}\right)\left(74.0 \frac{\mathrm{cm}^{3}}{\mathrm{~mol}}\right)}{\left((0.82)\left(74.0 \frac{\mathrm{cm}^{3}}{\mathrm{~mol}}\right)+(0.18)\left(71.3 \frac{\mathrm{cm}^{3}}{\mathrm{~mol}}\right)\right)}=71.8 \mathrm{~cm}^{3} / \mathrm{mol}
\end{aligned}
$$

Final calculation of estimated solubility parameter for PTMA in acetone:

$$
\begin{gathered}
\delta_{2}^{\prime}=\frac{\left(V_{m l}^{\frac{1}{2}} \delta_{m l}+V_{m h}^{\frac{1}{2}} \delta_{m h}\right)}{\left(V_{m l}^{\frac{1}{2}}+V_{m h}^{\frac{1}{2}}\right)}=\frac{\left(\left(89.4 \frac{\mathrm{cm}^{3}}{\mathrm{~mol}}\right)^{\frac{1}{2}} 17.2 \mathrm{MPa}^{\frac{1}{2}}+\left(71.8 \frac{\mathrm{cm}^{3}}{\mathrm{~mol}}\right)^{\frac{1}{2}} 28.0 \mathrm{MPa}^{\frac{1}{2}}\right)}{\left(\left(89.4 \frac{\mathrm{cm}^{3}}{\mathrm{~mol}}\right)^{\frac{1}{2}}+\left(71.8 \frac{\mathrm{cm}^{3}}{\mathrm{~mol}}\right)^{\frac{1}{2}}\right)} \\
\delta_{2}^{\prime}=22.3 \mathrm{MPa}^{\frac{1}{2}}
\end{gathered}
$$

Figure S18. Sample Calculation for PTMA-mCPBA in Acetone, as seen in Figure 3. 


\section{Calculation of Nitroxide and Oxoammonium Group Contribution Values Details}

To estimate the Hildebrand group contribution values for the nitroxide group, the molar volume contribution of the oxygen radical $(-\mathrm{O} \bullet)$ was assumed to be the same value as an oxygen (-O-) or $6.4 \mathrm{~cm}^{3} \cdot \mathrm{mol}^{-1}$ for the Hoy method and $3.8 \mathrm{~cm}^{3} \cdot \mathrm{mol}^{-1}$ for the Fedors method. This resulted in values of $1325.0 \mathrm{~J}^{1 / 2} \cdot \mathrm{cm}^{3 / 2} \cdot \mathrm{mol}^{-1}$ and $36.3 \mathrm{~kJ} \cdot \mathrm{mol}^{-1}$ for the Hoy and Fedors methods, respectively, for the oxygen radical $(-\mathrm{O} \bullet)$. The assumed molar volume and estimated group contribution values were combined with the known values for a tertiary amine to estimate the values for the entire nitroxide group (N-O•), Table 2.

To estimate the Hansen group contribution values for the nitroxide group, the same procedure was applied. The molar volume contribution of the oxygen radical $(-\mathrm{O} \bullet)$ was assumed to be the same value as an oxygen (-O-) or $3.8 \mathrm{~cm}^{3} \cdot \mathrm{mol}^{-1}$. Calculations for the oxygen radical resulted in $21 \mathrm{~J}^{1 / 2} \cdot \mathrm{cm}^{3 / 2} \cdot \mathrm{mol}^{-1}$ for $F_{\mathrm{d}}, 668 \mathrm{~J}^{1 / 2} \cdot \mathrm{cm}^{3 / 2} \cdot \mathrm{mol}^{-1}$ for $F_{\mathrm{p}}$, and $0 \mathrm{~J} \cdot \mathrm{mol}^{-1}$ for $U_{\mathrm{h}}$, which can be used to calculate $\delta_{d}, \delta_{p}$, and $\delta_{h}$, respectively. Solving for $F_{d}$ and $U_{h}$ would have resulted in 2003 $\mathrm{J}^{1 / 2} \cdot \mathrm{cm}^{3 / 2} \cdot \mathrm{mol}^{-1}$ and $-3476 \mathrm{~J} \cdot \mathrm{mol}^{-1}$, respectively, however, the $\mathrm{F}_{\mathrm{d}}$ value was much larger than those seen for other functional groups (largest of $1528 \mathrm{~J}^{1 / 2} \cdot \mathrm{cm}^{3 / 2} \cdot \mathrm{mol}^{-1}$ ), so the $F_{\mathrm{d}}$ was scaled (i.e. to a third of the calculated value). ${ }^{21,22}$ Additionally, only positive $U_{\mathrm{h}}$ values are assigned to functional groups capable of hydrogen bonding (for $-\mathrm{OH}, \mathrm{U}_{\mathrm{h}}=20000 \mathrm{~J} \cdot \mathrm{mol}^{-1}$ ) or $0 \mathrm{~J} \cdot \mathrm{mol}^{-1}$ for functional groups that do not hydrogen bond, so a value of $0 \mathrm{~J} \cdot \mathrm{mol}^{-1}$ was used for $\mathrm{U}_{\mathrm{h} \cdot{ }^{21}}$ Again, the assumed molar volume and estimated group contribution values were combined with the known values for a tertiary amine to estimate the values for the entire nitroxide group (N-O•), Table 2.

Back-calculation of the dispersion contribution $\left(\mathrm{F}_{\mathrm{d}}\right)$ for the oxoammonium cation would have resulted in $-4812 \mathrm{~J}^{1 / 2} \cdot \mathrm{cm}^{3 / 2} \cdot \mathrm{mol}^{-1}$, which is much larger than other functional groups so the $\mathrm{F}_{\mathrm{d}}$ was scaled to the largest value reported (i.e. $-409 \mathrm{~J}^{1 / 2} \cdot \mathrm{cm}^{3 / 2} \cdot \mathrm{mol}^{-1}$ ). ${ }^{21}$ Additionally, calculation of the 
polarity contribution $\left(\mathrm{F}_{\mathrm{p}}\right)$ for the oxoammonium cation would have resulted in $4254 \mathrm{~J}^{1 / 2} \cdot \mathrm{cm}^{3 / 2} \cdot \mathrm{mol}^{-}$ , which is much larger than other functional groups (largest of $1528 \mathrm{~J}^{1 / 2} \cdot \mathrm{cm}^{3 / 2} \cdot \mathrm{mol}^{-1}$ ), so the $F_{d}$ was scaled to be more similar (i.e. to a third of the calculated value). ${ }^{22}$ 


\section{For Hoy Method calculation of PTMA-mCPBA:}

$$
\begin{gathered}
\delta_{m C P B A, H o y}=21.1 \mathrm{MPa}^{1 / 2}(\text { from turbidity titrations }) \\
\delta_{m C P B A, \mathrm{Hoy}}=\left(\frac{0.36\left(F_{N H}\right)+051\left(F_{N O \bullet}\right)+0.13\left(F_{+N O}\right)}{0.36\left(V_{N H}\right)+0.51\left(V_{N O}\right)+0.13\left(V_{+N O}\right)}\right)=21.1 \mathrm{MPa}^{1 / 2}
\end{gathered}
$$

where $F$ is the group contribution sum and $V$ is the molar volume of a polymer with only the subscripted functional group. The value of $F_{N H}$ and $V_{N H}$ are known from published group contribution values. $V_{N O}$ and $V_{+N O}$ were estimated as described above.

$$
\delta_{m C P B A, \mathrm{Hoy}}=\left(\frac{3882.2+0.51\left(F_{N O}\right)+0.13\left(F_{+N O}\right)}{220.5}\right)=21.1 M P a^{1 / 2}
$$

For Hoy Method calculation of PTMA- $\mathrm{H}_{2} \mathrm{O}_{2}$ :

$$
\begin{gathered}
\delta_{H 2 O 2, \text { Hoy }}=21.7 \mathrm{MPa}^{1 / 2}(\text { from turbidity titrations }) \\
\delta_{H 2 O 2, \mathrm{Hoy}}=\left(\frac{0.27\left(F_{N H}\right)+0.67\left(F_{N O} \cdot\right)+0.06\left(F_{+N O}\right)}{0.27\left(V_{N H}\right)+0.67\left(V_{N O}\right)+0.06\left(V_{+N O}\right)}\right)=21.7 \mathrm{MPa}^{1 / 2} \\
\delta_{H 2 O 2, \text { Hoy }}=\left(\frac{3869.1+0.67\left(F_{N O} \cdot\right)+0.06\left(F_{+N O}\right)}{221.2}\right)=21.7 M P^{1 / 2}
\end{gathered}
$$

\section{Simplifying both expressions:}

PTMA- $m$ CPBA: $707.6=0.51 F_{N O}+0.13 F_{+N O}$

PTMA- $\mathrm{H}_{2} \mathrm{O}_{2}: 931.7=0.67 F_{N O} \cdot+0.06 F_{+N O}$

Solving for PTMA-mCPBA equation for $F_{+N O}$ :

$$
F_{+N O}=\frac{707.6-0.51 F_{N O} \bullet}{0.13}=5927.7-3.9 F_{N O}
$$

Plugging the expression for $F_{+N O}$ into PTMA- $\mathrm{H}_{2} \mathrm{O}_{2}$ equation to solve for $\boldsymbol{F}_{\mathrm{NO}}$.:

$$
\begin{gathered}
931.7=0.67 F_{N O \bullet}+0.06\left(5927.7-3.9 F_{N O}\right) \\
576.0=0.4 F_{N O} \bullet \\
F_{N O \bullet}=1325.0 \mathrm{~J}^{1 / 2} \cdot \mathrm{cm}^{3 / 2} \cdot \mathrm{mol}^{-1}
\end{gathered}
$$

Plugging $F_{N O}$. into PTMA-mCPBA equation to solve for $F_{+N O}$ :

$$
\begin{gathered}
707.6=0.51(1325.0)+0.13 F_{+N O} \\
F_{+N O}=243.5 \mathrm{~J}^{1 / 2} \cdot \mathrm{cm}^{3 / 2} \cdot \mathrm{mol}^{-1}
\end{gathered}
$$

Figure S19. Example calculation of the nitroxide and oxoammonium group contribution values (for the Hoy method) from experimental input. 
Table S8. Hydrogen bonding $\left(\delta_{\mathrm{h}}\right)$, polar $\left(\delta_{\mathrm{p}}\right)$, and dispersion $\left(\delta_{\mathrm{d}}\right)$ and Hansen solubility parameters for the polymers studied in this work, predicted values for other macromolecular radicals, commodity polymers, binder polymers, and conductive additives.

\begin{tabular}{|c|c|c|c|c|c|}
\hline Compound & Abbreviation & $\begin{array}{c}\delta_{h} \\
\left(\mathbf{M P a}^{1 / 2}\right)\end{array}$ & $\begin{array}{c}\delta_{\mathrm{p}} \\
\left(\mathrm{MPa}^{1 / 2}\right)\end{array}$ & $\begin{array}{c}\delta_{d} \\
\left(M^{1 / 2}\right)\end{array}$ & Reference \\
\hline $\mathrm{Ti}_{3} \mathrm{C}_{2} \mathrm{~T}_{\mathrm{x}}$ MXene & NA & 23.2 & 13.4 & 17.8 & 24,30 \\
\hline graphene oxide & $\mathrm{GO}$ & 15.7 & 10 & 17.1 & 24 \\
\hline Super P carbon black & Super P & 12.9 & 2.42 & 17.6 & 31 \\
\hline $\begin{array}{l}\operatorname{poly}(2,2,6,6- \\
\text { tetramethylpiperidine } \\
\text { methacrylate })\end{array}$ & PTMPM & 9.7 & 7.5 & 16.8 & This Work \\
\hline $\begin{array}{l}\text { poly(vinylidene } \\
\text { difluoride) }\end{array}$ & PVDF & 9.2 & 12.5 & 17.2 & 32 \\
\hline $\begin{array}{c}\text { poly(TEMPO } \\
\text { norbornene) }\end{array}$ & PTNB & 8.3 & 3.8 & 21.5 & $\begin{array}{l}\text { Predicted } \\
\text { from This } \\
\text { Work }\end{array}$ \\
\hline $\begin{array}{l}\text { poly(methyl } \\
\text { methacrylate) }\end{array}$ & PMMA & 7.8 & 5.7 & 17.5 & 21 \\
\hline graphene & - & 7.7 & 9.3 & 18 & 27 \\
\hline $\begin{array}{c}\text { poly(TEMPO ethylene } \\
\text { oxide) }\end{array}$ & PTEO & 7.7 & 7.1 & 19.6 & $\begin{array}{l}\text { Predicted } \\
\text { from This } \\
\text { Work }\end{array}$ \\
\hline $\begin{array}{c}\text { single-walled carbon } \\
\text { nanotubes }\end{array}$ & SWCNTs & 7.6 & 7.5 & 17.8 & $24,28,29$ \\
\hline reduced graphene oxide & rGO & 7.5 & 8.5 & 18 & 24 \\
\hline $\begin{array}{c}\text { poly(TEMPO vinyl } \\
\text { ether) }\end{array}$ & PTVE & 6.9 & 8.0 & 18.9 & $\begin{array}{l}\text { Predicted } \\
\text { from This } \\
\text { Work }\end{array}$ \\
\hline $\begin{array}{c}\text { poly(TEMPO } \\
\text { methacrylate) } m \text { CPBA } \\
\text { oxidation }\end{array}$ & $\begin{array}{l}\text { PTMA- } \\
m \mathrm{CPBA}\end{array}$ & 6.8 & 9.6 & 17.2 & This Work \\
\hline $\begin{array}{c}\text { poly(TEMPO } \\
\text { methacrylate) } \mathrm{H}_{2} \mathrm{O}_{2} \\
\text { oxidation }\end{array}$ & PTMA- $\mathrm{H}_{2} \mathrm{O}_{2}$ & 6.8 & 9.6 & 17.2 & This Work \\
\hline $\begin{array}{c}\text { poly(TEMPO } \\
\text { acrylamide })\end{array}$ & PTAm & 6.6 & 7.4 & 18.7 & $\begin{array}{l}\text { Predicted } \\
\text { from This } \\
\text { Work }\end{array}$ \\
\hline $\begin{array}{l}\text { multi-walled carbon } \\
\text { nanotubes }\end{array}$ & MWCNTs & 4.8 & 7 & 18.5 & 25,26 \\
\hline
\end{tabular}




\begin{tabular}{|c|c|c|c|c|c|}
\hline fullerene & C60 & 2.7 & 2.9 & 19.7 & 23,24 \\
\hline polystyrene & PS & 2.1 & 0.9 & 19.2 & 21 \\
\hline polyethylene & PE & 0.0 & 0.0 & 17.3 & 21 \\
\hline poly(tetrafluoroethylene) & PTFE & 0.0 & 0.0 & 12.7 & 21 \\
\hline
\end{tabular}

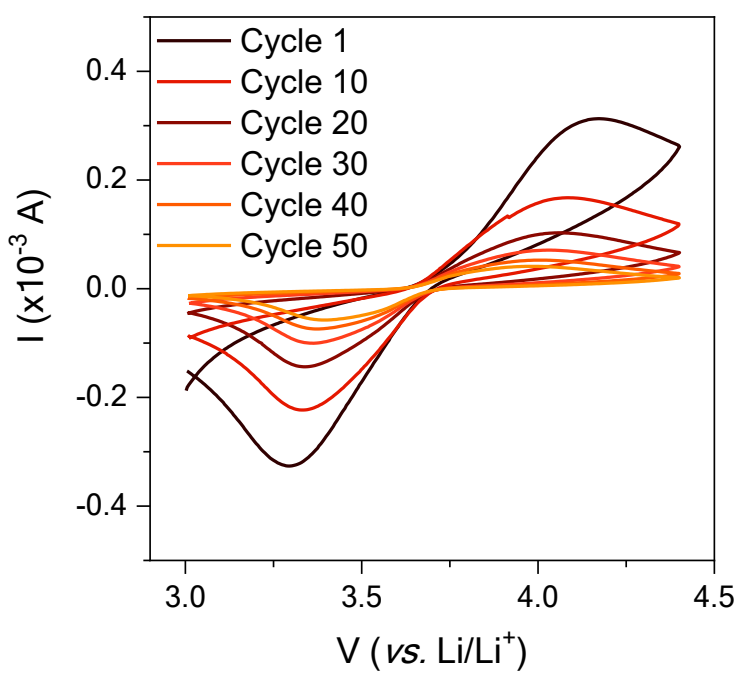

Figure S20. Cyclic voltammetry at $10 \mathrm{mV} \cdot \mathrm{s}^{-1}$ for a three-electrode beaker cell. The working electrode was a PTMA thin film on ITO-coated glass. The electrolyte was $0.5 \mathrm{M} \mathrm{LiCF} \mathrm{SO}_{3}$ in $\mathrm{EC} / \mathrm{DEC}(3 / 7, \mathrm{v} / \mathrm{v})$ and the counter and reference electrodes were lithium metal. 


\section{REFERENCES}

1. Janoschka, T.; Hager, M. D.; Schubert, U. S. Powering up the Future: Radical Polymers for Battery Applications. Advanced Materials 2012, 24 (48), 6397-6409 DOI: 10.1002/adma.201203119.

2. Nakahara, K.; Iwasa, S.; Satoh, M.; Morioka, Y.; Iriyama, J.; Suguro, M.; Hasegawa, E. Rechargeable batteries with organic radical cathodes. Chemical Physics Letters 2002, 359 (5), 351-354 DOI: https://doi.org/10.1016/S0009-2614(02)00705-4.

3. Deng, L.-f.; Li, X.-h.; Xiao, L.-X.; Zhang, Y.-h. Synthesis and electrochemical properties of polyradical cathode material for lithium second batteries. Journal of Central South University of Technology 2003, 10 (3), 190-194 DOI: 10.1007/s11771-003-0006-x.

4. $\quad$ Nishide, H.; Iwasa, S.; Pu, Y.-J.; Suga, T.; Nakahara, K.; Satoh, M. Organic radical battery: nitroxide polymers as a cathode-active material. Electrochimica Acta 2004, 50 (2), 827-831 DOI: https://doi.org/10.1016/j.electacta.2004.02.052.

5. Nakahara, K.; Iriyama, J.; Iwasa, S.; Suguro, M.; Satoh, M.; Cairns, E. J. Al-laminated film packaged organic radical battery for high-power applications. Journal of Power Sources 2007, 163 (2), 1110-1113 DOI: https://doi.org/10.1016/j.jpowsour.2006.10.003.

6. Nakahara, K.; Iriyama, J.; Iwasa, S.; Suguro, M.; Satoh, M.; Cairns, E. J. High-rate capable organic radical cathodes for lithium rechargeable batteries. Journal of Power Sources 2007, 165 (2), 870-873 DOI: https://doi.org/10.1016/j.jpowsour.2006.11.045.

7. Nakahara, K.; Iriyama, J.; Iwasa, S.; Suguro, M.; Satoh, M.; Cairns, E. J. Cell properties for modified PTMA cathodes of organic radical batteries. Journal of Power Sources 2007, 165 (1), 398-402 DOI: https://doi.org/10.1016/j.jpowsour.2006.11.044.

8. Kim, J.-K.; Cheruvally, G.; Choi, J.-W.; Ahn, J.-H.; Choi, D. S.; Song, C. E. Rechargeable Organic Radical Battery with Electrospun, Fibrous Membrane-Based Polymer Electrolyte. Journal of The Electrochemical Society 2007, 154 (9), A839 DOI: 10.1149/1.2752022.

9. Kim, J.-K.; Cheruvally, G.; Choi, J.-W.; Ahn, J.-H.; Lee, S. H.; Choi, D. S.; Song, C. E. Effect of radical polymer cathode thickness on the electrochemical performance of organic radical battery. Solid State Ionics 2007, 178 (27), 1546-1551 DOI: https://doi.org/10.1016/j.ssi.2007.09.009.

10. Bugnon, L.; Morton, C. J. H.; Novak, P.; Vetter, J.; Nesvadba, P. Synthesis of Poly(4methacryloyloxy-TEMPO) via Group-Transfer Polymerization and Its Evaluation in Organic Radical Battery. Chemistry of Materials 2007, 19 (11), 2910-2914 DOI: 10.1021/cm063052h.

11. Kim, J.-K.; Cheruvally, G.; Ahn, J.-H.; Seo, Y.-G.; Choi, D. S.; Lee, S.-H.; Song, C. E. Organic radical battery with PTMA cathode: Effect of PTMA content on electrochemical properties. Journal of Industrial and Engineering Chemistry 2008, 14 (3), 371-376 DOI: https://doi.org/10.1016/i.jiec.2007.12.002.

12. Kim, J.-K.; Ahn, J.-H.; Cheruvally, G.; Chauhan, G. S.; Choi, J.-W.; Kim, D.-S.; Ahn, H.-J.; Lee, S. H.; Song, C. E. Electrochemical properties of rechargeable organic radical battery with PTMA cathode. Metals and Materials International 2009, 15 (1), 77-82 DOI: 10.1007/s12540-009-0077-9.

13. Lin, H.-C.; Li, C.-C.; Lee, J.-T. Nitroxide polymer brushes grafted onto silica nanoparticles as cathodes for organic radical batteries. Journal of Power Sources 2011, 196 (19), 8098-8103 DOI: https://doi.org/10.1016/j.jpowsour.2011.05.037.

14. Guo, W.; Yin, Y.-X.; Xin, S.; Guo, Y.-G.; Wan, L.-J. Superior radical polymer cathode material with a two-electron process redox reaction promoted by graphene. Energy \& Environmental Science 2012, 5 (1), 5221-5225 DOI: 10.1039/C1EE02148F.

15. Kim, Y.; Jo, C.; Lee, J.; Lee, C. W.; Yoon, S. An ordered nanocomposite of organic radical polymer and mesocellular carbon foam as cathode material in lithium ion batteries. Journal of Materials Chemistry 2012, 22 (4), 1453-1458 DOI: 10.1039/C1JM15053G.

16. Hung, M.-K.; Wang, Y.-H.; Lin, C.-H.; Lin, H.-C.; Lee, J.-T. Synthesis and electrochemical behaviour of nitroxide polymer brush thin-film electrodes for organic radical batteries. Journal of Materials Chemistry 2012, 22 (4), 1570-1577 DOI: 10.1039/C1JM13911H. 
17. Zhang, K.; Hu, Y.; Wang, L.; Fan, J.; Monteiro, M. J.; Jia, Z. The impact of the molecular weight on the electrochemical properties of poly(TEMPO methacrylate). Polymer Chemistry 2017, 8 (11), 1815 1823 DOI: $10.1039 /$ C7PY00151G.

18. Wang, S.; Li, F.; Easley, A. D.; Lutkenhaus, J. L. Real-time insight into the doping mechanism of redox-active organic radical polymers. Nature Materials 2019, 18 (1), 69-75 DOI: 10.1038/s41563-0180215-1.

19. Wang, S.; Park, A. M. G.; Flouda, P.; Easley, A. D.; Li, F.; Ma, T.; Fuchs, G. D.; Lutkenhaus, J. L. Solution-Processable Thermally Crosslinked Organic Radical Polymer Battery Cathodes. ChemSusChem 2020, n/a (n/a), DOI: 10.1002/cssc.201903554.

20. Xue, W.; Mutlu, H.; Theato, P. Post-polymerization modification of polymeric active esters towards TEMPO containing polymers: A systematic study. European Polymer Journal 2020, 130, 109660 DOI: https://doi.org/10.1016/j.eurpolymj.2020.109660.

21. Barton, A. F. M., CRC Handbook of Solubility Parameters and Other Cohesion Parameters, Second Edition. Taylor \& Francis: 1991.

22. Hansen, C. M. The three dimensional solubility parameter. Danish Technical: Copenhagen 1967,

14.

23. Hansen, C. M.; Smith, A. L. Using Hansen solubility parameters to correlate solubility of C60 fullerene in organic solvents and in polymers. Carbon 2004, 42 (8), 1591-1597 DOI: https://doi.org/10.1016/j.carbon.2004.02.011.

24. Qin, J.; Wang, X.; Jiang, Q.; Cao, M. Optimizing Dispersion, Exfoliation, Synthesis, and Device Fabrication of Inorganic Nanomaterials Using Hansen Solubility Parameters. ChemPhysChem 2019, 20 (9), 1069-1097 DOI: 10.1002/cphc.201900110.

25. Clark, M. D.; Krishnamoorti, R. Dispersion of Functionalized Multiwalled Carbon Nanotubes. The Journal of Physical Chemistry C 2009, 113 (49), 20861-20868 DOI: 10.1021/jp907221g.

26. detriche, S.; Zorzini, G.; Colomer, J.-F.; Fonseca, A.; B.Nagy, J. Application of the Hansen Solubility Parameters Theory to Carbon Nanotubes. Journal of nanoscience and nanotechnology 2008, 8, 6082-92 DOI: 10.1166/jnn.2008.SW16.

27. Hernandez, Y.; Lotya, M.; Rickard, D.; Bergin, S. D.; Coleman, J. N. Measurement of Multicomponent Solubility Parameters for Graphene Facilitates Solvent Discovery. Langmuir 2010, 26 (5), 3208-3213 DOI: 10.1021/la903188a.

28. Ata, S.; Mizuno, T.; Nishizawa, A.; Subramaniam, C.; Futaba, D. N.; Hata, K. Influence of matching solubility parameter of polymer matrix and CNT on electrical conductivity of CNT/rubber composite. Scientific Reports 2014, 4 (1), 7232 DOI: 10.1038/srep07232.

29. Bergin, S. D.; Nicolosi, V.; Streich, P. V.; Giordani, S.; Sun, Z.; Windle, A. H.; Ryan, P.; Niraj, N. P. P.; Wang, Z.-T. T.; Carpenter, L.; Blau, W. J.; Boland, J. J.; Hamilton, J. P.; Coleman, J. N. Towards Solutions of Single-Walled Carbon Nanotubes in Common Solvents. Advanced Materials 2008, 20 (10), 1876-1881 DOI: 10.1002/adma.200702451.

30. Maleski, K.; Mochalin, V. N.; Gogotsi, Y. Dispersions of Two-Dimensional Titanium Carbide MXene in Organic Solvents. Chemistry of Materials 2017, 29 (4), 1632-1640 DOI: 10.1021/acs.chemmater.6b04830.

31. Kwon, Y. H.; Huie, M. M.; Choi, D.; Chang, M.; Marschilok, A. C.; Takeuchi, K. J.; Takeuchi, E. S.; Reichmanis, E. Toward Uniformly Dispersed Battery Electrode Composite Materials: Characteristics and Performance. ACS Applied Materials \& Interfaces 2016, 8 (5), 3452-3463 DOI: 10.1021/acsami.5b11938.

32. Bottino, A.; Capannelli, G.; Munari, S.; Turturro, A. Solubility parameters of poly(vinylidene fluoride). Journal of Polymer Science Part B: Polymer Physics 1988, 26 (4), 785-794 DOI: 10.1002/polb.1988.090260405. 